\title{
Search for Narrow Resonances Decaying to Dijets in Proton-Proton Collisions at $\sqrt{s}=13 \mathrm{TeV}$
}

V. Khachatryan et al. ${ }^{*}$

(CMS Collaboration)

(Received 3 December 2015; published 18 February 2016)

\begin{abstract}
A search for narrow resonances in proton-proton collisions at $\sqrt{s}=13 \mathrm{TeV}$ is presented. The invariant mass distribution of the two leading jets is measured with the CMS detector using a data set corresponding to an integrated luminosity of $2.4 \mathrm{fb}^{-1}$. The highest observed dijet mass is $6.1 \mathrm{TeV}$. The distribution is smooth and no evidence for resonant particles is observed. Upper limits at $95 \%$ confidence level are set on the production cross section for narrow resonances with masses above $1.5 \mathrm{TeV}$. When interpreted in the context of specific models, the limits exclude string resonances with masses below 7.0 TeV, scalar diquarks below $6.0 \mathrm{TeV}$, axigluons and colorons below $5.1 \mathrm{TeV}$, excited quarks below $5.0 \mathrm{TeV}$, color-octet scalars below $3.1 \mathrm{TeV}$, and $W^{\prime}$ bosons below $2.6 \mathrm{TeV}$. These results significantly extend previously published limits.
\end{abstract}

DOI: 10.1103/PhysRevLett.116.071801

Deep inelastic proton-proton $(p p)$ collisions often produce two or more energetic jets when the constituent partons are scattered with large transverse momenta $\left(p_{\mathrm{T}}\right)$. The invariant mass $m_{\mathrm{jj}}$ of the pair of jets having the largest values of $p_{\mathrm{T}}$ in the event (the dijet) has a spectrum that is predicted by quantum chromodynamics (QCD) to fall steeply and smoothly with increasing dijet mass $[1,2]$. Many extensions of the standard model predict the existence of new massive particles that couple to quarks $(q)$ and gluons $(g)$ and can be detected as resonances in the dijet mass spectrum. In this Letter, we report a search for narrow resonances, those with natural widths that are small compared to the experimental resolution. The search uses the dijet mass spectrum measured with the CMS detector at a center-of-mass energy of $\sqrt{s}=13 \mathrm{TeV}$. The data correspond to an integrated luminosity of $2.4 \mathrm{fb}^{-1}$ from the 2015 running of the CERN LHC (Run 2).

The most stringent current bounds on dijet resonance production have been presented by the CMS [3-7] and ATLAS [8-12] Collaborations, using proton-proton collisions at $\sqrt{s}=7$ and $8 \mathrm{TeV}$ from LHC Run 1. A summary of previous searches and a comparison of the different strategies are presented in Ref. [13]. The present search is expected to be more sensitive than previous studies for dijet masses above $2 \mathrm{TeV}$, and has similar sensitivity as a contemporaneous search from ATLAS at $\sqrt{s}=13 \mathrm{TeV}$ [14]. We present a model-independent search and, in addition, consider the following models of $s$-channel dijet

*Full author list given at the end of the article.

Published by the American Physical Society under the terms of the Creative Commons Attribution 3.0 License. Further distribution of this work must maintain attribution to the author(s) and the published article's title, journal citation, and DOI. resonances: string resonances [15,16], scalar diquarks [17], axigluons $[18,19]$, colorons $[19,20]$, excited quarks $\left(q^{*}\right)$ [21,22], color-octet scalars [23], new gauge bosons ( $W^{\prime}$ and $Z^{\prime}$ ) [24], and Randall-Sundrum (RS) gravitons (G) [25]. More details on the specific choices of couplings for these models can be found in Ref. [5].

The CMS detector and its coordinate system, including the azimuthal angle $\phi$ (in radians) and the pseudorapidity $\eta$, are described in detail in Ref. [26]. Events are selected using a two-tier trigger system. Events satisfying loose jet requirements at the first level (L1) are examined by the high-level trigger (HLT), where jets are clustered from particle-flow (PF) $[27,28]$ candidates, discussed in the next paragraph. The jets with $p_{\mathrm{T}}>40 \mathrm{GeV}$ and $|\eta|<3$ are used to compute $H_{\mathrm{T}}$, the scalar sum of the jet $p_{T}$. Events are accepted if they have $H_{T}>800 \mathrm{GeV}$ or include a jet with $p_{T}>500 \mathrm{GeV}$. At least one reconstructed vertex is required with $|z|<24 \mathrm{~cm}$. The primary vertex is defined as the vertex with the highest sum of $p_{\mathrm{T}}^{2}$ of the associated tracks.

The PF algorithm is used to reconstruct the particles in an event and to identify them as muons, electrons (with associated bremsstrahlung photons), photons (unconverted and converted into $e^{+} e^{-}$pairs), and either charged or neutral hadrons. These PF candidates are clustered into jets using the anti- $k_{t}$ algorithm [29] with a distance parameter of 0.4, implemented in the FastJet package [30]. Charged $\mathrm{PF}$ candidates not originating from the primary vertex are removed prior to the jet finding. An event-by-event jet-area-based correction [31-33] is applied to the jets to remove the estimated energy from additional collisions in the same or adjacent bunch crossings (pileup). The jet momenta and energies are further corrected using calibration constants obtained from simulation, test beam results, and $p p$ collision data at $\sqrt{s}=13 \mathrm{TeV}$, using methods 
described in Ref. [33] with all in situ calibrations obtained from the current data. All jets are required to have $p_{\mathrm{T}}>$ $30 \mathrm{GeV}$ and $|\eta|<2.5$. The two jets with largest $p_{\mathrm{T}}$ are defined as the leading jets. Jet identification criteria [34] are applied to remove spurious jets associated with calorimeter noise. An event is rejected if either of the two leading jets does not satisfy the jet identification criteria.

Geometrically close jets are combined into "wide jets" and used to determine the dijet mass, as in our previous searches [4-7]. The wide-jet algorithm, designed for dijet resonance event reconstruction, reduces the analysis sensitivity to gluon radiation from the final state partons. The two leading jets are used as seeds and the four-vectors of all other jets, if within $\Delta R=\sqrt{(\Delta \eta)^{2}+(\Delta \phi)^{2}}<1.1$, are added to the nearest leading jet to obtain two wide jets, which then form the dijet system. The background from $t$-channel dijet events is suppressed by requiring the pseudorapidity separation of the two wide jets to satisfy $\left|\Delta \eta_{\mathrm{jj}}\right|<1.3$. The above requirements, originally developed for the analysis of Run 1 data, maximize the search sensitivity for isotropic decays of dijet resonances in the presence of QCD dijet background. It has been verified that these requirements remain optimal for collisions at $\sqrt{s}=13 \mathrm{TeV}$. We select events with $m_{\mathrm{jj}}>1.2 \mathrm{TeV}$ for which the combined L1 trigger and HLT are found to be fully efficient.

Figure 1 shows the dijet mass spectrum, defined as the observed number of events in each bin divided by the integrated luminosity and bin width, with predefined bins of width corresponding to the dijet mass resolution [3]. The highest dijet mass observed is $6.1 \mathrm{TeV}$. The data are compared with a leading-order QCD Monte Carlo (MC) prediction from the PYTHIA 8 (v205) [35] generator with the CUETP8M1 tune [36,37], including a GEANT4-based [38] simulation of the CMS detector. The PYTHIA simulation uses the NNPDF2.3LO [39] parton distribution functions (PDF). The renormalization and factorization scales are both set at the $p_{\mathrm{T}}$ value of the hard-scattered partons. The $\mathrm{MC}$ prediction is normalized to the integrated contents of the data in Fig. 1, requiring multiplication of the predicted cross section by a factor of 0.88 .

To test the smoothness of the measured dijet mass spectrum, we fit the data with the parametrization

$$
\frac{d \sigma}{d m_{\mathrm{jj}}}=\frac{P_{0}(1-x)^{P_{1}}}{x^{P_{2}+P_{3} \ln (x)}},
$$

where $x=m_{\mathrm{jj}} / \sqrt{s}$ and $P_{0}, P_{1}, P_{2}$, and $P_{3}$ are fitted parameters. This functional form was also used in previous searches $[3-12,40]$ to describe the data and the QCD predictions. In Fig. 1 we show the result of the binned maximum likelihood fit, which yields $\chi^{2}=31$ for 35 degrees of freedom, where the $\chi^{2}$ is determined using the Poisson uncertainties shown in Fig. 1. The data are seen to be well described by the fit.

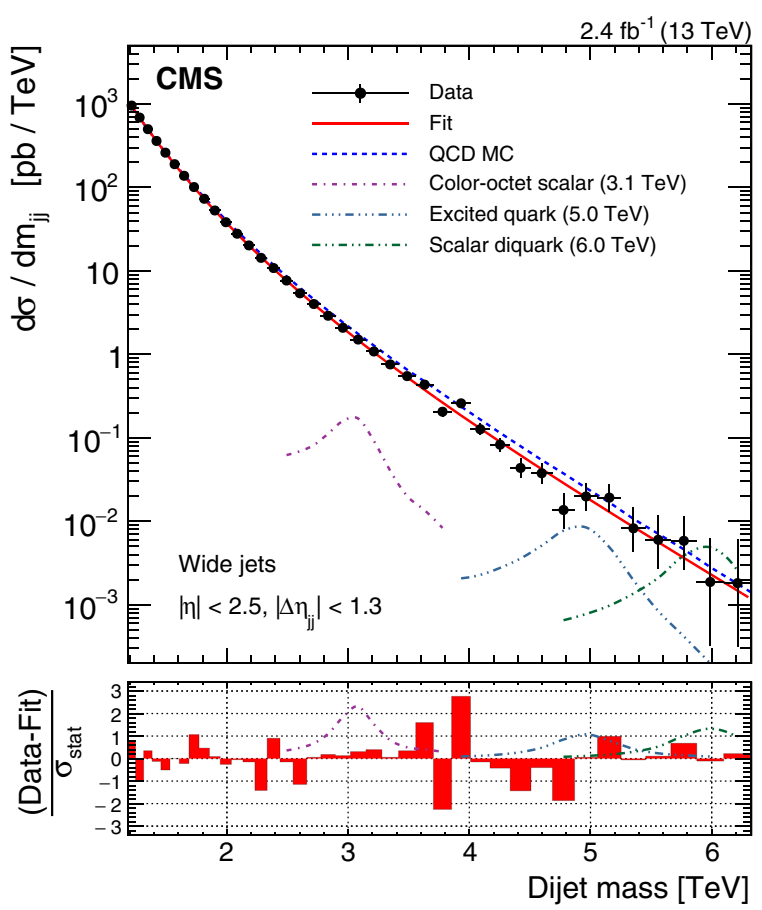

FIG. 1. Dijet mass spectrum (points) compared to a fitted parametrization (solid curve) and to the prediction of the PYTHIA 8 [35] QCD MC event generator including simulation of the detector (dashed curve). The lower panel shows the difference between the data and the fitted parametrization, divided by the statistical uncertainties. The predicted distributions of narrow resonance signals for three models, with resonance mass values corresponding to the respective $95 \%$ confidence level exclusion limit, are shown in both panels (dash-dotted curves).

We search in the dijet mass spectrum for narrow resonances. Figure 2 shows example dijet mass distributions for simulated signal events, generated with the PYTHIA 8 program. The predicted mass distributions have Gaussian cores from the jet energy resolution, and tails towards lower mass values primarily from QCD radiation. The contribution of this low-mass tail to the line shape depends on the parton content of the resonance ( $q q, q g$, or $g g$ ). Resonances containing gluons, which emit QCD radiation more strongly than quarks, have a more pronounced tail. For the high-mass resonances, there is also a significant contribution that depends both on the PDF and on the natural width of the Breit-Wigner resonance. For resonances produced through interactions of nonvalent partons in the proton, the low-mass component of the Breit-Wigner resonance distribution is amplified by the rise of the parton probability distribution at low fractional momentum. These effects cause a large tail at low mass values. Neglecting the tails, the approximate value of the dijet mass resolution varies with resonance mass from $7 \%$ at $1.5 \mathrm{TeV}$ to $4 \%$ at $7 \mathrm{TeV}$.

There is no evidence for a narrow resonance in the data, as seen from Fig. 1. The most significant excess in the data relative to the background fit occurs for a dijet mass of 


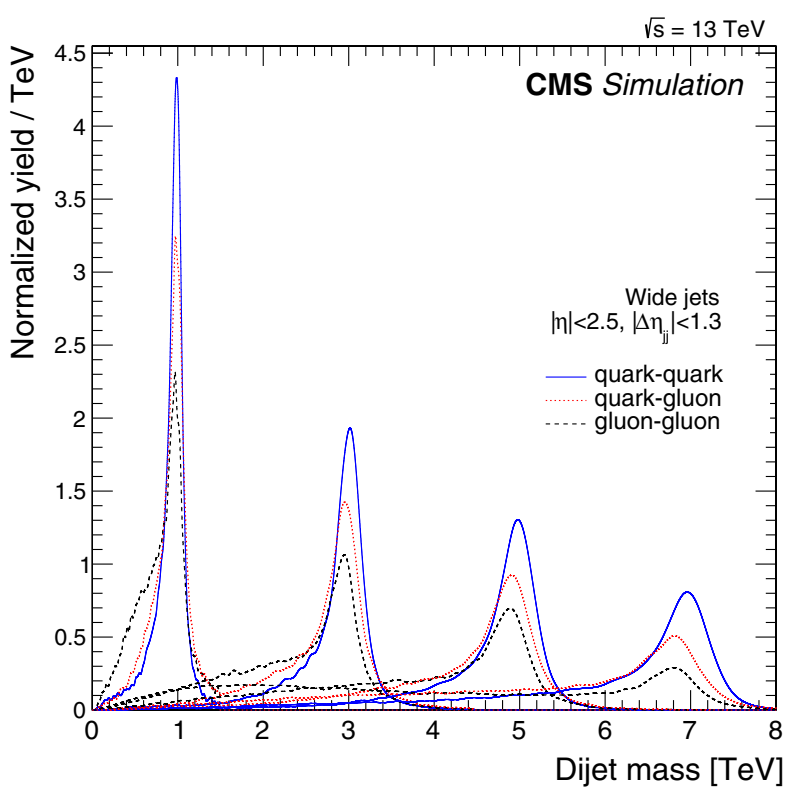

FIG. 2. The reconstructed resonance mass spectrum predicted by the PYTHIA 8 [35] MC event generator, including simulation of the detector, for resonances generated with mass values $1,3,5$, and $7 \mathrm{TeV}$, for quark-quark processes modeled by $q \bar{q} \rightarrow G \rightarrow q \bar{q}$ (solid line), for quark-gluon processes modeled by $q g \rightarrow q^{*} \rightarrow$ $q g$ (dotted line), and for gluon-gluon processes modeled by $g g \rightarrow G \rightarrow g g$ (dashed line), where $G$ is a $\mathrm{RS}$ graviton and $q^{*}$ is an excited quark.

3.9 TeV. A fit to the hypothesis of a narrow $q q$ resonance, which includes contributions from the bin at $3.9 \mathrm{TeV}$ and neighboring bins, has a local statistical significance of 1.7 standard deviations. Figure 1 includes example signal distributions of the three kinds of narrow resonances $(q q, q g$, and $g g)$ at the mass values $(6.0,5.0$, and $3.1 \mathrm{TeV})$ corresponding to the limit set on the respective models (scalar diquark, excited quark, and color-octet scalar). These limits are presented below.

We use the dijet mass spectrum from wide jets, the background parametrization, and the dijet resonance shapes to set limits on new particles decaying to the parton pairs $q q$ (or $q \bar{q}$ ), $q g$, and $g g$. A separate limit is determined for each final state $(q q, q g$, and $g g)$ because of the dependence of the dijet resonance shape on the type of the two final-state partons.

The dominant sources of systematic uncertainty are the jet energy scale, jet energy resolution, integrated luminosity, and the estimation of background. The uncertainty in the jet energy scale is $2 \%$, determined from Run 2 data using the methods described in Ref. [33]. This uncertainty is propagated to the limits by shifting the dijet mass for signal events by $\pm 2 \%$. The uncertainty in the jet energy resolution translates into an uncertainty of $10 \%$ in the resolution of the dijet mass [33], and is propagated to the limits by increasing and decreasing by $10 \%$ the reconstructed width of the dijet mass shape for signal. The luminosity scale and its uncertainty are estimated from beam-beam scans utilizing the methods from Ref. [41]. The uncertainty in the integrated luminosity is $12 \%$, and is propagated to the normalization of the signal. Not included in this analysis is a recent reevaluation of the integrated luminosity that increases the measured value by $4.3 \%$ and reduces the systematic uncertainty to $4.6 \%$. Changes in the values of the parameters describing the background introduce a change in the signal strength that is accounted for as a systematic uncertainty. The dependence of the signal mass distributions on the number of pileup interactions is negligible.

To set upper limits on signal cross sections we use a Bayesian formalism [42] with a uniform prior for a positive signal cross section; log-normal priors are used to model systematic uncertainties in the jet energy scale, jet energy resolution, and integrated luminosity, all treated as nuisance parameters to be integrated over. We calculate the likelihood, namely the posterior probability density as a function of resonance cross section, independently at each value of resonance pole mass from 1.5 to $7.2 \mathrm{TeV}$ in $0.1 \mathrm{TeV}$ steps. Resonances with masses less than $1.5 \mathrm{TeV}$ are too close to the lower edge of our dijet mass spectrum to produce a peak distinguishable from the background and are therefore not considered. Examples of the resonance shapes used are shown in Fig. 2. The data are fitted with the background function plus a signal shape, with the signal cross section a fitted parameter. The resulting fitting function, with the signal cross section set to zero, is used as the background estimate. The likelihood is formed using as input the data, the background estimate from the best fit of the signal + background hypothesis to the data, and the resonance shape multiplied by the resonance cross section. The uncertainty in the background is incorporated through marginalization, i.e., by integrating the likelihood over the background parameters using uniform priors. The integration is performed for each of the background nuisance parameters in a range around the best-fit values, corresponding to a decrease in the likelihood by a factor of 1000 from its maximum value for each parameter independently.

Figure 3 shows the model-independent observed upper limits at $95 \%$ confidence level (C.L.) on $\sigma B A$, i.e., the product of the cross section $(\sigma)$, the branching fraction $(B)$, and the acceptance $(A)$ for the kinematic requirements $\left|\Delta \eta_{\mathrm{jj}}\right|<1.3$ and $|\eta|<2.5$, for narrow $q q, q g$, and $g g$ resonances. The acceptance of the requirement $m_{\mathrm{jj}}>$ 1.2 $\mathrm{TeV}$ has been taken into account by correcting the limits, and therefore does not appear in the acceptance $A$. Figure 3 also shows the expected limits on the cross section and their bands of uncertainty. The expected limits are estimated with pseudoexperiments generated using background-only hypotheses. The generated mass spectra are fit with a background + signal model to extract expected upper limits. The difference in the limits for $q q, q g$, and $g g$ resonances at the same resonance mass originates from the difference in their line shapes. All upper limits presented 

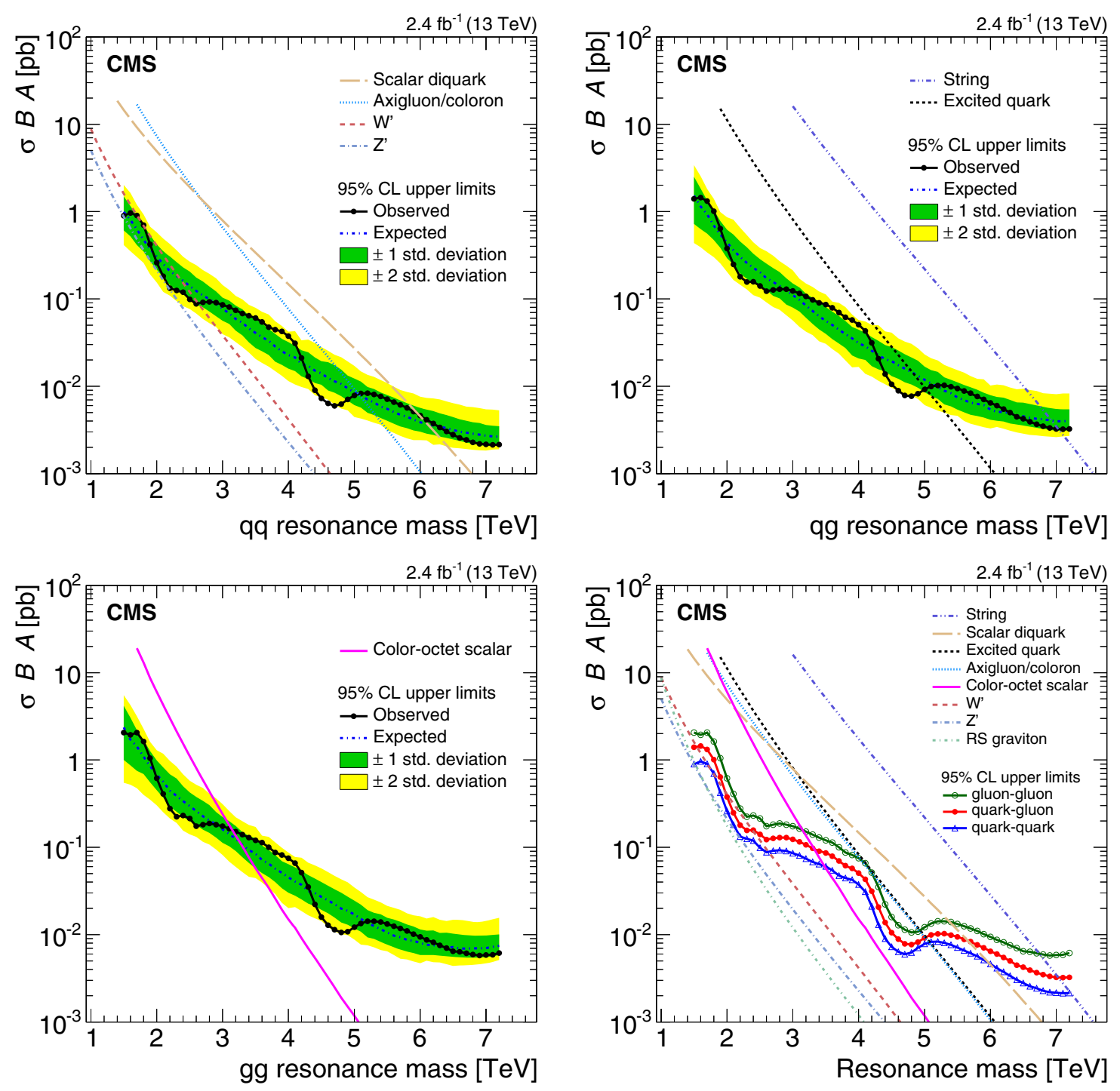

FIG. 3. The observed 95\% CL upper limits on the product of the cross section, branching fraction, and acceptance for quark-quark (top left), quark-gluon (top right), and gluon-gluon (bottom left) type dijet resonances, shown as symbols connected by solid curves, and a comparison of all types (bottom right). The corresponding expected limits (dash-dotted curves) and their variation at the 1 and 2 standard deviation levels (shaded bands) are also shown. The limits are compared to the predicted cross sections of string resonances [15,16], scalar diquarks [17], excited quarks [21,22], axigluons [18,19], colorons [19,20], color-octet scalars [23], new gauge bosons $W^{\prime}$ and $Z^{\prime}$ [24], and RS gravitons [25].

are compared to the parton-level predictions of $\sigma B A$, without detector simulation, to determine mass limits on new particles. The model predictions shown in Fig. 3 are calculated in the narrow-width approximation [13] using the CTEQ6L1 [43] PDF at leading order, with a next-toleading order correction factor of approximately 1.3 included for the $W^{\prime}$ and $Z^{\prime}$ models [44], and approximately 1.2 for the axigluon and coloron models [19]. The branching fraction $B$ includes the direct decays of the resonance into $q q, q g$, and $g g$ only, excluding top quarks, and no other decays giving jets are considered in the theoretical calculation of the model cross section. The acceptance is evaluated at the parton level for the resonance decay to two partons. In the case of isotropic decays it is $A \approx 0.6$ independent of resonance mass.

For a given model, new particles are excluded at $95 \% \mathrm{CL}$ in mass regions where the theoretical prediction lies at or above the observed upper limit for the appropriate final state of Fig. 3. The mass limits reported in Table I represent significant extensions of the most stringent observed limits from LHC Run 1 [7,12]. For string resonances, the observed mass limit of 7.0 TeV extends the previous CMS limit of $5.0 \mathrm{TeV}$; for scalar diquarks, the observed mass limit of 6.0 TeV extends the previous CMS limit of 4.7 $\mathrm{TeV}$; for axigluons and colorons, the observed mass limit of 5.1 TeV extends the previous CMS limit of $3.6 \mathrm{TeV}$; 
TABLE I. Observed and expected mass limits for analyses that exclude the listed models at 95\% CL for a resonance mass from $1.5 \mathrm{TeV}$ up to the indicated values.

\begin{tabular}{lccc}
\hline \hline Model & $\begin{array}{c}\text { Final } \\
\text { state }\end{array}$ & $\begin{array}{c}\text { Observed mass } \\
\text { limit }[\mathrm{TeV}]\end{array}$ & $\begin{array}{c}\text { Expected mass } \\
\text { limit }[\mathrm{TeV}]\end{array}$ \\
\hline String & $q g$ & 7.0 & 6.9 \\
Scalar diquark & $q q$ & 6.0 & 6.1 \\
Axigluon/coloron & $q \bar{q}$ & 5.1 & 5.1 \\
Excited quark $\left(q^{*}\right)$ & $q g$ & 5.0 & 4.8 \\
Color-octet scalar & $g g$ & 3.1 & 3.3 \\
Heavy $W\left(W^{\prime}\right)$ & $q \bar{q}$ & 2.6 & 2.3 \\
\hline \hline
\end{tabular}

for excited quarks, we set a mass limit of 5.0 TeV compared to the ATLAS limit of $4.06 \mathrm{TeV}$; for a color-octet scalar, the observed mass limit of $3.1 \mathrm{TeV}$ improves the ATLAS limit of $2.70 \mathrm{TeV}$; and for a $W^{\prime}$ boson, we exclude masses up to $2.6 \mathrm{TeV}$, just beyond the ATLAS limit of $2.45 \mathrm{TeV}$. With the current data sample we cannot set mass limits on $Z^{\prime}$ bosons with standard-model-like couplings or on RS gravitons with dimensionless coupling less than 0.1 .

In summary, a search for narrow resonances decaying into a pair of jets has been performed using a data sample of $p p$ collisions at $\sqrt{s}=13 \mathrm{TeV}$ corresponding to an integrated luminosity of $2.4 \mathrm{fb}^{-1}$. The dijet mass spectrum has been measured to be a smoothly falling distribution. In the analyzed data sample, there is no evidence for resonant particle production. We present generic upper limits on the product $\sigma B A$ that are applicable to any model of narrow dijet resonance production. This search is more sensitive than previous searches for dijet resonances for masses above $2 \mathrm{TeV}$. We set the most stringent limits to date on the masses of string resonances, scalar diquarks, axigluons, colorons, excited quarks, color-octet scalars, and $W^{\prime}$ bosons.

We congratulate our colleagues in the CERN accelerator departments for the excellent performance of the LHC and thank the technical and administrative staffs at CERN and at other CMS institutes for their contributions to the success of the CMS effort. In addition, we gratefully acknowledge the computing centers and personnel of the Worldwide LHC Computing Grid for delivering so effectively the computing infrastructure essential to our analyses. Finally, we acknowledge the enduring support for the construction and operation of the LHC and the CMS detector provided by the following funding agencies: BMWFW and FWF (Austria); FNRS and FWO (Belgium); CNPq, CAPES, FAPERJ, and FAPESP (Brazil); MES (Bulgaria); CERN; CAS, MoST, and NSFC (China); COLCIENCIAS (Colombia); MSES and CSF (Croatia); RPF (Cyprus); MoER, ERC IUT and ERDF (Estonia); Academy of Finland, MEC, and HIP (Finland); CEA and CNRS/IN2P3 (France); BMBF, DFG, and HGF (Germany); GSRT (Greece); OTKA and NIH (Hungary); DAE and DST (India); IPM (Iran); SFI (Ireland); INFN (Italy); MSIP and NRF (Republic of Korea); LAS (Lithuania); MOE and UM (Malaysia); CINVESTAV, CONACYT, SEP, and UASLP-FAI (Mexico); MBIE (New Zealand); PAEC (Pakistan); MSHE and NSC (Poland); FCT (Portugal); JINR (Dubna); MON, RosAtom, RAS and RFBR (Russia); MESTD (Serbia); SEIDI and CPAN (Spain); Swiss Funding Agencies (Switzerland); MST (Taipei); ThEPCenter, IPST, STAR and NSTDA (Thailand); TUBITAK and TAEK (Turkey); NASU and SFFR (Ukraine); STFC (United Kingdom); DOE and NSF (USA).

[1] CMS Collaboration, Measurement of the differential dijet production cross section in proton-proton collisions at $\sqrt{s}=7 \mathrm{TeV}$, Phys. Lett. B 700, 187 (2011).

[2] ATLAS Collaboration, Measurement of dijet cross sections in $p p$ collisions at $7 \mathrm{TeV}$ centre-of-mass energy using the ATLAS detector, J. High Energy Phys. 05 (2014) 059.

[3] CMS Collaboration, Search for Dijet Resonances in $7 \mathrm{TeV}$ p p Collisions at CMS, Phys. Rev. Lett. 105, 211801 (2010).

[4] CMS Collaboration, Search for resonances in the dijet mass spectrum from $7 \mathrm{TeV} p p$ collisions at CMS, Phys. Lett. B 704, 123 (2011).

[5] CMS Collaboration, Search for narrow resonances and quantum black holes in inclusive and $b$-tagged dijet mass spectra from $p p$ collisions at $\sqrt{s}=7 \mathrm{TeV}$, J. High Energy Phys. 01 (2013) 013.

[6] CMS Collaboration, Search for narrow resonances using the dijet mass spectrum in $p p$ collisions at $\sqrt{s}=8 \mathrm{TeV}$, Phys. Rev. D 87, 114015 (2013).

[7] CMS Collaboration, Search for resonances and quantum black holes using dijet mass spectra in proton-proton collisions at $\sqrt{s}=8 \mathrm{TeV}$, Phys. Rev. D 91, 052009 (2015).

[8] ATLAS Collaboration, Search for New Particles in Two-Jet Final States in $7 \mathrm{TeV}$ Proton-Proton Collisions with the ATLAS Detector at the LHC, Phys. Rev. Lett. 105, 161801 (2010).

[9] ATLAS Collaboration, Search for new physics in dijet mass and angular distributions in $p p$ collisions at $\sqrt{s}=7 \mathrm{TeV}$ measured with the ATLAS detector, New J. Phys. 13, 053044 (2011).

[10] ATLAS Collaboration, Search for new physics in the dijet mass distribution using $1 \mathrm{fb}^{-1}$ of $p p$ collision data at $\sqrt{s}=7 \mathrm{TeV}$ collected by the ATLAS detector, Phys. Lett. B 708, 37 (2012).

[11] ATLAS Collaboration, ATLAS search for new phenomena in dijet mass and angular distributions using $p p$ collisions at $\sqrt{s}=7 \mathrm{TeV}$, J. High Energy Phys. 01 (2013) 029.

[12] ATLAS Collaboration, Search for new phenomena in the dijet mass distribution using $p p$ collision data at $\sqrt{s}=8 \mathrm{TeV}$ with the ATLAS detector, Phys. Rev. D 91, 052007 (2015).

[13] R. M. Harris and K. Kousouris, Searches for dijet resonances at hadron colliders, Int. J. Mod. Phys. A 26, 5005 (2011).

[14] ATLAS Collaboration, Search for new phenomena in dijet mass and angular distributions from $p p$ collisions at $\sqrt{s}=13 \mathrm{TeV}$ with the ATLAS detector, arXiv:1512.01530. 
[15] L. A. Anchordoqui, H. Goldberg, D. Lüst, S. Nawata, S. Stieberger, and T. R. Taylor, Dijet Signals for Low Mass Strings at the LHC, Phys. Rev. Lett. 101, 241803 (2008).

[16] S. Cullen, M. Perelstein, and M. E. Peskin, TeV strings and collider probes of large extra dimensions, Phys. Rev. D 62, 055012 (2000).

[17] J. L. Hewett and T. G. Rizzo, Low-energy phenomenology of superstring-inspired $E_{6}$ models, Phys. Rep. 183, 193 (1989).

[18] P. H. Frampton and S. L. Glashow, Chiral color: An alternative to the standard model, Phys. Lett. B 190, 157 (1987).

[19] R. S. Chivukula, E. H. Simmons, A. Farzinnia, and J. Ren, Hadron collider production of massive color-octet vector bosons at next-to-leading order, Phys. Rev. D 87, 094011 (2013).

[20] E. H. Simmons, Coloron phenomenology, Phys. Rev. D 55, 1678 (1997).

[21] U. Baur, I. Hinchliffe, and D. Zeppenfeld, Excited quark production at hadron colliders, Int. J. Mod. Phys. A 02, 1285 (1987).

[22] U. Baur, M. Spira, and P. M. Zerwas, Excited quark and lepton production at hadron colliders, Phys. Rev. D 42, 815 (1990).

[23] T. Han, I. Lewis, and Z. Liu, Colored resonant signals at the LHC: Largest rate and simplest topology, J. High Energy Phys. 12 (2010) 085.

[24] E. Eichten, I. Hinchliffe, K. D. Lane, and C. Quigg, Supercollider physics, Rev. Mod. Phys. 56, 579 (1984).

[25] L. Randall and R. Sundrum, An Alternative to Compactification, Phys. Rev. Lett. 83, 4690 (1999).

[26] CMS Collaboration, The CMS experiment at the CERN LHC, J. Instrum. 3, S08004 (2008).

[27] CMS Collaboration, CMS Physics Analysis Summary Report No. CMS-PAS-PFT-09-001, 2009; http://cdsweb .cern.ch/record/1194487.

[28] CMS Collaboration, CMS Physics Analysis Summary Report No. CMS-PAS-PFT-10-001, 2010; http://cdsweb .cern.ch/record/1247373.

[29] M. Cacciari, G. P. Salam, and G. Soyez, The anti- $k_{t}$ jet clustering algorithm, J. High Energy Phys. 04 (2008) 063.
[30] M. Cacciari and G. P. Salam, Dispelling the $N^{3}$ myth for the $k_{t}$ jet-finder, Phys. Lett. B 641, 57 (2006).

[31] M. Cacciari, G. P. Salam, and G. Soyez, FastJet user manual, Eur. Phys. J. C 72, 1896 (2012).

[32] M. Cacciari and G. P. Salam, Pileup subtraction using jet areas, Phys. Lett. B 659, 119 (2008).

[33] CMS Collaboration, Determination of jet energy calibration and transverse momentum resolution in CMS, J. Instrum. 6, P11002 (2011).

[34] CMS Collaboration, CMS Physics Analysis Summary Report No. CMS-PAS-JME-10-003, 2010; http://cdsweb .cern.ch/record/1279362.

[35] T. Sjöstrand, S. Mrenna, and P. Skands, A brief introduction to PYTHIA 8.1, Comput. Phys. Commun. 178, 852 (2008).

[36] CMS Collaboration, Event generator tunes obtained from underlying event and multiparton scattering measurements, arXiv:1512.00815.

[37] P. Skands, S. Carrazza, and J. Rojo, Tuning PYTHIA 8.1: The Monash 2013 tune, Eur. Phys. J. C 74, 3024 (2014).

[38] S. Agostinelli et al. (GEANT4 Collaboration), GEANT4A simulation toolkit, Nucl. Instrum. Methods Phys. Res., Sect. A 506, 250 (2003).

[39] R. D. Ball, V. Bertone, S. Carrazza, C. S. Deans, L. Del Debbio, S. Forte, A. Guffanti, N. P. Hartland, J. I. Latorre, J. Rojo, and M. Ubiali (NNPDF), Parton distributions with LHC data, Nucl. Phys. B867, 244 (2013).

[40] T. Aaltonen et al. (CDF Collaboration), Search for new particles decaying into dijets in proton-antiproton collisions at $\sqrt{s}=1.96 \mathrm{TeV}$, Phys. Rev. D 79, 112002 (2009).

[41] CMS Collaboration, CMS Physics Analysis Summary Report No. CMS-PAS-LUM-13-001, 2013; http://cdsweb .cern.ch/record/1598864.

[42] K. A. Olive et al. (Particle Data Group), Review of particle physics, Chin. Phys. C 38, 090001 (2014).

[43] J. Pumplin, D. R. Stump, J. Huston, H.-L. Lai, P. Nadolsky, and W.-K. Tung, New generation of parton distributions with uncertainties from global QCD analysis, J. High Energy Phys. 07 (2002) 012.

[44] K. Gumus, N. Akchurin, S. Esen, and R. M Harris, Report No. CMS-NOTE-2006-070, 2006; https://cds.cern.ch/ record/962025.

V. Khachatryan, ${ }^{1}$ A. M. Sirunyan, ${ }^{1}$ A. Tumasyan, ${ }^{1}$ W. Adam, ${ }^{2}$ E. Asilar, ${ }^{2}$ T. Bergauer, ${ }^{2}$ J. Brandstetter, ${ }^{2}$ E. Brondolin, ${ }^{2}$ M. Dragicevic, ${ }^{2}$ J. Erö, ${ }^{2}$ M. Flechl, ${ }^{2}$ M. Friedl, ${ }^{2}$ R. Frühwirth, ${ }^{2, b}$ V. M. Ghete, ${ }^{2}$ C. Hartl, ${ }^{2}$ N. Hörmann, ${ }^{2}$ J. Hrubec, ${ }^{2}$ M. Jeitler, ${ }^{2, b}$ V. Knünz, ${ }^{2}$ A. König, ${ }^{2}$ M. Krammer, ${ }^{2, b}$ I. Krätschmer, ${ }^{2}$ D. Liko, ${ }^{2}$ T. Matsushita, ${ }^{2}$ I. Mikulec, ${ }^{2}$ D. Rabady, ${ }^{2, c}$ B. Rahbaran, ${ }^{2}$ H. Rohringer, ${ }^{2}$ J. Schieck, ${ }^{2, b}$ R. Schöfbeck, ${ }^{2}$ J. Strauss, ${ }^{2}$ W. Treberer-Treberspurg, ${ }^{2}$ W. Waltenberger, ${ }^{2}$ C.-E. Wulz, ${ }^{2, b}$ V. Mossolov, ${ }^{3}$ N. Shumeiko, ${ }^{3}$ J. Suarez Gonzalez, ${ }^{3}$ S. Alderweireldt, ${ }^{4}$ T. Cornelis, ${ }^{4}$ E. A. De Wolf, ${ }^{4}$ X. Janssen, ${ }^{4}$ A. Knutsson, ${ }^{4}$ J. Lauwers, ${ }^{4}$ S. Luyckx, ${ }^{4}$ M. Van De Klundert, ${ }^{4}$ H. Van Haevermaet, ${ }^{4}$ P. Van Mechelen, ${ }^{4}$ N. Van Remortel, ${ }^{4}$ A. Van Spilbeeck, ${ }^{4}$ S. Abu Zeid, ${ }^{5}$ F. Blekman, ${ }^{5}$ J. D’Hondt, ${ }^{5}$ N. Daci, ${ }^{5}$ I. De Bruyn, ${ }^{5}$ K. Deroover, ${ }^{5}$ N. Heracleous, ${ }^{5}$ J. Keaveney, ${ }^{5}$ S. Lowette, ${ }^{5}$ L. Moreels, ${ }^{5}$ A. Olbrechts, ${ }^{5}$ Q. Python, ${ }^{5}$ D. Strom, ${ }^{5}$ S. Tavernier, ${ }^{5}$

W. Van Doninck, ${ }^{5}$ P. Van Mulders, ${ }^{5}$ G. P. Van Onsem, ${ }^{5}$ I. Van Parijs, ${ }^{5}$ P. Barria, ${ }^{6}$ H. Brun, ${ }^{6}$ C. Caillol, ${ }^{6}$ B. Clerbaux, ${ }^{6}$ G. De Lentdecker, ${ }^{6}$ G. Fasanella, ${ }^{6}$ L. Favart, ${ }^{6}$ R. Goldouzian, ${ }^{6}$ A. Grebenyuk, ${ }^{6}$ G. Karapostoli, ${ }^{6}$ T. Lenzi, ${ }^{6}$ A. Léonard, ${ }^{6}$ T. Maerschalk, ${ }^{6}$ A. Marinov, ${ }^{6}$ L. Perniè,${ }^{6}$ A. Randle-conde, ${ }^{6}$ T. Seva, ${ }^{6}$ C. Vander Velde,${ }^{6}$ P. Vanlaer, ${ }^{6}$ R. Yonamine, ${ }^{6}$ F. Zenoni, ${ }^{6}$ F. Zhang, ${ }^{6, d}$ K. Beernaert, ${ }^{7}$ L. Benucci, ${ }^{7}$ A. Cimmino, ${ }^{7}$ S. Crucy, ${ }^{7}$ D. Dobur, ${ }^{7}$ A. Fagot, ${ }^{7}$ G. Garcia, ${ }^{7}$ M. Gul, ${ }^{7}$ 
J. Mccartin, ${ }^{7}$ A. A. Ocampo Rios, ${ }^{7}$ D. Poyraz, ${ }^{7}$ D. Ryckbosch, ${ }^{7}$ S. Salva, ${ }^{7}$ M. Sigamani, ${ }^{7}$ M. Tytgat, ${ }^{7}$ W. Van Driessche, ${ }^{7}$ E. Yazgan, ${ }^{7}$ N. Zaganidis, ${ }^{7}$ S. Basegmez, ${ }^{8}$ C. Beluffi,${ }^{8, e}$ O. Bondu, ${ }^{8}$ S. Brochet, ${ }^{8}$ G. Bruno, ${ }^{8}$ A. Caudron, ${ }^{8}$ L. Ceard, ${ }^{8}$ C. Delaere, ${ }^{8}$ D. Favart, ${ }^{8}$ L. Forthomme, ${ }^{8}$ A. Giammanco, ${ }^{8, f}$ A. Jafari, ${ }^{8}$ P. Jez,${ }^{8}$ M. Komm, ${ }^{8}$ V. Lemaitre,${ }^{8}$ A. Mertens, ${ }^{8}$ M. Musich ${ }^{8}$ C. Nuttens,${ }^{8}$ L. Perrini, ${ }^{8}$ K. Piotrzkowski, ${ }^{8}$ A. Popov, ${ }^{8, g}$ L. Quertenmont, ${ }^{8}$ M. Selvaggi, ${ }^{8}$ M. Vidal Marono, ${ }^{8}$ N. Beliy, ${ }^{9}$ G. H. Hammad, ${ }^{9}$ W. L. Aldá Júnior, ${ }^{10}$ F. L. Alves ${ }^{10}$ G. A. Alves, ${ }^{10}$ L. Brito, ${ }^{10}$ M. Correa Martins Junior,${ }^{10}$ M. Hamer, ${ }^{10}$ C. Hensel, ${ }^{10}$ A. Moraes,${ }^{10}$ M. E. Pol, ${ }^{10}$ P. Rebello Teles, ${ }^{10}$ E. Belchior Batista Das Chagas, ${ }^{11}$ W. Carvalho, ${ }^{11}$ J. Chinellato, ${ }^{11, \mathrm{~h}}$ A. Custódio, ${ }^{11}$ E. M. Da Costa,${ }^{11}$ D. De Jesus Damiao, ${ }^{11}$ C. De Oliveira Martins,${ }^{11}$ S. Fonseca De Souza, ${ }^{11}$ L. M. Huertas Guativa, ${ }^{11}$ H. Malbouisson, ${ }^{11}$ D. Matos Figueiredo, ${ }^{11}$ C. Mora Herrera, ${ }^{11}$ L. Mundim, ${ }^{11}$ H. Nogima, ${ }^{11}$ W. L. Prado Da Silva, ${ }^{11}$ A. Santoro, ${ }^{11}$ A. Sznajder, ${ }^{11}$ E. J. Tonelli Manganote, ${ }^{11, h}$ A. Vilela Pereira, ${ }^{11}$ S. Ahuja, ${ }^{12 a}$ C. A. Bernardes, ${ }^{12 b}$ A. De Souza Santos, ${ }^{12 b}$ S. Dogra, ${ }^{12 a}$ T. R. Fernandez Perez Tomei, ${ }^{12 a}$ E. M. Gregores, ${ }^{12 b}$ P. G. Mercadante, ${ }^{12 b}$ C. S. Moon, ${ }^{12 a, i}$ S. F. Novaes, ${ }^{12 a}$ Sandra S. Padula, ${ }^{12 a}$ D. Romero Abad, ${ }^{12 a}$ J. C. Ruiz Vargas, ${ }^{12 a}$ A. Aleksandrov, ${ }^{13}$ R. Hadjiiska, ${ }^{13}$ P. Iaydjiev, ${ }^{13}$ M. Rodozov, ${ }^{13}$ S. Stoykova, ${ }^{13}$ G. Sultanov, ${ }^{13}$ M. Vutova,${ }^{13}$ A. Dimitrov, ${ }^{14}$ I. Glushkov, ${ }^{14}$ L. Litov, ${ }^{14}$ B. Pavlov, ${ }^{14}$ P. Petkov, ${ }^{14}$ M. Ahmad, ${ }^{15}$ J. G. Bian, ${ }^{15}$ G. M. Chen, ${ }^{15}$ H. S. Chen, ${ }^{15}$ M. Chen, ${ }^{15}$ T. Cheng, ${ }^{15}$ R. Du, ${ }^{15}$ C. H. Jiang, ${ }^{15}$ R. Plestina, ${ }^{15, j}$ F. Romeo, ${ }^{15}$ S. M. Shaheen, ${ }^{15}$ A. Spiezia, ${ }^{15}$ J. Tao, ${ }^{15}$ C. Wang, ${ }^{15}$ Z. Wang, ${ }^{15}$ H. Zhang, ${ }^{15}$ C. Asawatangtrakuldee, ${ }^{16}$ Y. Ban, ${ }^{16}$ Q. Li,${ }^{16}$ S. Liu, ${ }^{16}$ Y. Mao, ${ }^{16}$ S. J. Qian, ${ }^{16}$ D. Wang, ${ }^{16}$ Z. Xu, ${ }^{16}$ C. Avila, ${ }^{17}$ A. Cabrera, ${ }^{17}$ L. F. Chaparro Sierra, ${ }^{17}$ C. Florez,${ }^{17}$ J. P. Gomez,${ }^{17}$ B. Gomez Moreno, ${ }^{17}$ J. C. Sanabria, ${ }^{17}$ N. Godinovic, ${ }^{18}$ D. Lelas, ${ }^{18}$ I. Puljak, ${ }^{18}$ P. M. Ribeiro Cipriano, ${ }^{18}$ Z. Antunovic, ${ }^{19}$ M. Kovac, ${ }^{19}$ V. Brigljevic, ${ }^{20}$ K. Kadija ${ }^{20}$ J. Luetic, ${ }^{20}$ S. Micanovic, ${ }^{20}$ L. Sudic,${ }^{20}$ A. Attikis,${ }^{21}$ G. Mavromanolakis, ${ }^{21}$ J. Mousa, ${ }^{21}$ C. Nicolaou, ${ }^{21}$ F. Ptochos, ${ }^{21}$ P. A. Razis, ${ }^{21}$ H. Rykaczewski, ${ }^{21}$ M. Bodlak, ${ }^{22}$ M. Finger, ${ }^{22, \mathrm{k}}$ M. Finger Jr., ${ }^{22, \mathrm{k}}$ Y. Assran, ${ }^{23,1, \mathrm{~m}}$ S. Elgammal, ${ }^{23,1}$ A. Ellithi Kamel, ${ }^{23, n, n}$ M. A. Mahmoud ${ }^{23,0,0}$ B. Calpas, ${ }^{24}$ M. Kadastik, ${ }^{24}$ M. Murumaa, ${ }^{24}$ M. Raidal, ${ }^{24}$ A. Tiko, ${ }^{24}$ C. Veelken, ${ }^{24}$ P. Eerola, ${ }^{25}$ J. Pekkanen, ${ }^{25}$ M. Voutilainen ${ }^{25}$ J. Härkönen ${ }^{26}$ V. Karimäki,${ }^{26}$ R. Kinnunen, ${ }^{26}$ T. Lampén, ${ }^{26}$ K. Lassila-Perini, ${ }^{26} \mathrm{~S}$. Lehti, ${ }^{26}$ T. Lindén, ${ }^{26}$ P. Luukka, ${ }^{26}$ T. Peltola, ${ }^{26}$ E. Tuominen, ${ }^{26} \mathrm{~J}$. Tuominiemi, ${ }^{26}$ E. Tuovinen, ${ }^{26}$

L. Wendland, ${ }^{26}$ J. Talvitie, ${ }^{27}$ T. Tuuva, ${ }^{27}$ M. Besancon, ${ }^{28}$ F. Couderc, ${ }^{28}$ M. Dejardin, ${ }^{28}$ D. Denegri, ${ }^{28}$ B. Fabbro, ${ }^{28}$ J. L. Faure, ${ }^{28}$ C. Favaro, ${ }^{28}$ F. Ferri, ${ }^{28}$ S. Ganjour, ${ }^{28}$ A. Givernaud ${ }^{28}$ P. Gras,${ }^{28}$ G. Hamel de Monchenault,${ }^{28}$ P. Jarry, ${ }^{28}$ E. Locci, ${ }^{28}$ M. Machet, ${ }^{28}$ J. Malcles, ${ }^{28}$ J. Rander, ${ }^{28}$ A. Rosowsky, ${ }^{28}$ M. Titov, ${ }^{28}$ A. Zghiche, ${ }^{28}$ I. Antropov, ${ }^{29}$ S. Baffioni, ${ }^{29}$ F. Beaudette, ${ }^{29}$ P. Busson, ${ }^{29}$ L. Cadamuro, ${ }^{29}$ E. Chapon, ${ }^{29}$ C. Charlot, ${ }^{29}$ O. Davignon, ${ }^{29}$ N. Filipovic, ${ }^{29}$

R. Granier de Cassagnac, ${ }^{29}$ M. Jo, ${ }^{29}$ S. Lisniak, ${ }^{29}$ L. Mastrolorenzo, ${ }^{29}$ P. Miné ${ }^{29}$ I. N. Naranjo, ${ }^{29}$ M. Nguyen, ${ }^{29}$ C. Ochando ${ }^{29}$ G. Ortona, ${ }^{29}$ P. Paganini, ${ }^{29}$ P. Pigard, ${ }^{29}$ S. Regnard, ${ }^{29}$ R. Salerno, ${ }^{29}$ J. B. Sauvan, ${ }^{29}$ Y. Sirois,${ }^{29}$ T. Strebler,${ }^{29}$ Y. Yilmaz, ${ }^{29}$ A. Zabi, ${ }^{29}$ J.-L. Agram, ${ }^{30, p}$ J. Andrea, ${ }^{30}$ A. Aubin, ${ }^{30}$ D. Bloch,${ }^{30}$ J.-M. Brom, ${ }^{30}$ M. Buttignol, ${ }^{30}$ E. C. Chabert,${ }^{30}$ N. Chanon, ${ }^{30}$ C. Collard, ${ }^{30}$ E. Conte, ${ }^{30, p}$ X. Coubez, ${ }^{30}$ J.-C. Fontaine, ${ }^{30, p}$ D. Gelé, ${ }^{30}$ U. Goerlach, ${ }^{30}$ C. Goetzmann, ${ }^{30}$ A.-C. Le Bihan, ${ }^{30}$ J. A. Merlin, ${ }^{30, c}$ K. Skovpen, ${ }^{30}$ P. Van Hove, ${ }^{30}$ S. Gadrat,${ }^{31}$ S. Beauceron, ${ }^{32}$ C. Bernet, ${ }^{32}$ G. Boudoul, ${ }^{32}$ E. Bouvier, ${ }^{32}$ C. A. Carrillo Montoya,${ }^{32}$ R. Chierici, ${ }^{32}$ D. Contardo, ${ }^{32}$ B. Courbon, ${ }^{32}$ P. Depasse, ${ }^{32}$ H. El Mamouni, ${ }^{32}$ J. Fan, ${ }^{32}$ J. Fay, ${ }^{32}$ S. Gascon, ${ }^{32}$ M. Gouzevitch, ${ }^{32}$ B. Ille, ${ }^{32}$ F. Lagarde, ${ }^{32}$ I. B. Laktineh, ${ }^{32}$ M. Lethuillier, ${ }^{32}$ L. Mirabito ${ }^{32}$ A. L. Pequegnot, ${ }^{32}$ S. Perries, ${ }^{32}$ J. D. Ruiz Alvarez, ${ }^{32}$ D. Sabes, ${ }^{32}$ L. Sgandurra,${ }^{32}$ V. Sordini, ${ }^{32}$ M. Vander Donckt, ${ }^{32}$ P. Verdier, ${ }^{32}$ S. Viret, ${ }^{32}$ T. Toriashvili, ${ }^{33, q}$ L. Rurua,${ }^{34}$ C. Autermann, ${ }^{35}$ S. Beranek,${ }^{35}$ L. Feld, ${ }^{35}$ A. Heister, ${ }^{35}$ M. K. Kiesel, ${ }^{35}$ K. Klein, ${ }^{35}$ M. Lipinski, ${ }^{35}$ A. Ostapchuk,${ }^{35}$ M. Preuten, ${ }^{35}$ F. Raupach,,${ }^{35}$ S. Schael, ${ }^{35}$ J. F. Schulte, ${ }^{35}$ T. Verlage,${ }^{35}$ H. Weber,${ }^{35}$ V. Zhukov, ${ }^{35,9}$ M. Ata, ${ }^{36}$ M. Brodski, ${ }^{36}$ E. Dietz-Laursonn, ${ }^{36}$ D. Duchardt, ${ }^{36}$ M. Endres, ${ }^{36}$ M. Erdmann, ${ }^{36}$ S. Erdweg, ${ }^{36}$

T. Esch, ${ }^{36}$ R. Fischer, ${ }^{36}$ A. Güth, ${ }^{36}$ T. Hebbeker, ${ }^{36}$ C. Heidemann, ${ }^{36}$ K. Hoepfner, ${ }^{36}$ S. Knutzen, ${ }^{36}$ P. Kreuzer ${ }^{36}$ M. Merschmeyer, ${ }^{36}$ A. Meyer, ${ }^{36}$ P. Millet ${ }^{36}$ S. Mukherjee, ${ }^{36}$ M. Olschewski, ${ }^{36}$ K. Padeken, ${ }^{36}$ P. Papacz, ${ }^{36}$ T. Pook, ${ }^{36}$ M. Radziej, ${ }^{36}$ H. Reithler, ${ }^{36}$ M. Rieger, ${ }^{36}$ F. Scheuch, ${ }^{36}$ L. Sonnenschein, ${ }^{36}$ D. Teyssier, ${ }^{36}$ S. Thüer, ${ }^{36}$ V. Cherepanov, ${ }^{37}$ Y. Erdogan, ${ }^{37}$ G. Flügge,${ }^{37}$ H. Geenen, ${ }^{37}$ M. Geisler, ${ }^{37}$ F. Hoehle,${ }^{37}$ B. Kargoll, ${ }^{37}$ T. Kress, ${ }^{37}$ A. Künsken, ${ }^{37}$ J. Lingemann, ${ }^{37}$ A. Nehrkorn, ${ }^{37}$ A. Nowack, ${ }^{37}$ I. M. Nugent,${ }^{37}$ C. Pistone, ${ }^{37}$ O. Pooth,${ }^{37}$ A. Stahl,${ }^{37}$ M. Aldaya Martin, ${ }^{38}$ I. Asin, ${ }^{38}$ N. Bartosik, ${ }^{38}$ O. Behnke, ${ }^{38}$ U. Behrens,${ }^{38}$ K. Borras ${ }^{38, r}$ A. Burgmeier,${ }^{38}$ A. Campbell, ${ }^{38}$ C. Contreras-Campana, ${ }^{38}$ F. Costanza, ${ }^{38}$ C. Diez Pardos, ${ }^{38}$ G. Dolinska, ${ }^{38}$ S. Dooling, ${ }^{38}$ T. Dorland ${ }^{38}$ G. Eckerlin, ${ }^{38}$ D. Eckstein, ${ }^{38}$ T. Eichhorn, ${ }^{38}$ G. Flucke, ${ }^{38}$ E. Gallo, ${ }^{38, s}$ J. Garay Garcia, ${ }^{38}$ A. Geiser, ${ }^{38}$ A. Gizhko, ${ }^{38}$ P. Gunnellini, ${ }^{38}$ J. Hauk,${ }^{38}$ M. Hempel,${ }^{38, t}$ H. Jung,${ }^{38}$ A. Kalogeropoulos, ${ }^{38}$ O. Karacheban, ${ }^{38, t}$ M. Kasemann, ${ }^{38}$ P. Katsas, ${ }^{38}$ J. Kieseler,${ }^{38}$ C. Kleinwort, ${ }^{38}$ I. Korol, ${ }^{38}$ W. Lange, ${ }^{38}$ J. Leonard, ${ }^{38}$ K. Lipka, ${ }^{38}$ A. Lobanov, ${ }^{38}$ W. Lohmann,${ }^{38, t}$ R. Mankel,${ }^{38}$ I.-A. Melzer-Pellmann, ${ }^{38}$ A. B. Meyer, ${ }^{38}$ G. Mittag, ${ }^{38}$ J. Mnich, ${ }^{38}$ A. Mussgiller, ${ }^{38}$ S. Naumann-Emme, ${ }^{38}$ A. Nayak, ${ }^{38}$ E. Ntomari, ${ }^{38}$ H. Perrey, ${ }^{38}$ D. Pitzl, ${ }^{38}$ R. Placakyte, ${ }^{38}$ A. Raspereza, ${ }^{38}$ B. Roland, ${ }^{38}$ M. Ö. Sahin, ${ }^{38}$ P. Saxena, ${ }^{38}$ T. Schoerner-Sadenius, ${ }^{38}$ C. Seitz, ${ }^{38}$ S. Spannagel, ${ }^{38}$ 
K. D. Trippkewitz ${ }^{38}$ R. Walsh, ${ }^{38}$ C. Wissing, ${ }^{38}$ V. Blobel, ${ }^{39}$ M. Centis Vignali, ${ }^{39}$ A. R. Draeger, ${ }^{39}$ J. Erfle, ${ }^{39}$ E. Garutti, ${ }^{39}$ K. Goebel, ${ }^{39}$ D. Gonzalez, ${ }^{39}$ M. Görner, ${ }^{39}$ J. Haller, ${ }^{39}$ M. Hoffmann, ${ }^{39}$ R. S. Höing, ${ }^{39}$ A. Junkes,${ }^{39}$ R. Klanner, ${ }^{39}$ R. Kogler, ${ }^{39}$ N. Kovalchuk, ${ }^{39}$ T. Lapsien, ${ }^{39}$ T. Lenz,${ }^{39}$ I. Marchesini, ${ }^{39}$ D. Marconi, ${ }^{39}$ M. Meyer, ${ }^{39}$ D. Nowatschin, ${ }^{39}$ J. Ott, ${ }^{39}$ F. Pantaleo, ${ }^{39, c}$ T. Peiffer, ${ }^{39}$ A. Perieanu, ${ }^{39}$ N. Pietsch,,${ }^{39}$ J. Poehlsen, ${ }^{39}$ D. Rathjens, ${ }^{39}$ C. Sander,${ }^{39}$ C. Scharf, ${ }^{39}$ P. Schleper,${ }^{39}$ E. Schlieckau, ${ }^{39}$ A. Schmidt, ${ }^{39}$ S. Schumann, ${ }^{39}$ J. Schwandt, ${ }^{39}$ V. Sola, ${ }^{39}$ H. Stadie, ${ }^{39}$ G. Steinbrück, ${ }^{39}$ F. M. Stober, ${ }^{39}$ H. Tholen, ${ }^{39}$ D. Troendle, ${ }^{39}$ E. Usai ${ }^{39}$ L. Vanelderen, ${ }^{39}$ A. Vanhoefer, ${ }^{39}$ B. Vormwald,${ }^{39}$ C. Barth,${ }^{40}$ C. Baus,${ }^{40}$ J. Berger, ${ }^{40}$ C. Böser ${ }^{40}$ E. Butz ${ }^{40}$ T. Chwalek,${ }^{40}$ F. Colombo, ${ }^{40}$ W. De Boer, ${ }^{40}$ A. Descroix ${ }^{40}$ A. Dierlamm, ${ }^{40}$ S. Fink,${ }^{40}$ F. Frensch, ${ }^{40}$ R. Friese, ${ }^{40}$ M. Giffels, ${ }^{40}$ A. Gilbert, ${ }^{40}$ D. Haitz ${ }^{40}$ F. Hartmann, ${ }^{40, c}$ S. M. Heindl, ${ }^{40}$ U. Husemann, ${ }^{40}$ I. Katkov, ${ }^{40, g}$ A. Kornmayer ${ }^{40, c}$ P. Lobelle Pardo, ${ }^{40}$ B. Maier ${ }^{40}$ H. Mildner, ${ }^{40}$ M. U. Mozer ${ }^{40}$ T. Müller, ${ }^{40}$ Th. Müller, ${ }^{40}$ M. Plagge, ${ }^{40}$ G. Quast, ${ }^{40}$ K. Rabbertz, ${ }^{40}$ S. Röcker, ${ }^{40}$ F. Roscher, ${ }^{40}$ M. Schröder, ${ }^{40}$ G. Sieber ${ }^{40}$ H. J. Simonis,${ }^{40}$ R. Ulrich, ${ }^{40}$ J. Wagner-Kuhr, ${ }^{40} \mathrm{~S}$. Wayand, ${ }^{40} \mathrm{M}$. Weber, ${ }^{40} \mathrm{~T}$. Weiler, ${ }^{40} \mathrm{~S}$. Williamson, ${ }^{40} \mathrm{C}$. Wöhrmann, ${ }^{40} \mathrm{R}$. Wolf, ${ }^{40} \mathrm{G}$. Anagnostou, ${ }^{41}$ G. Daskalakis, ${ }^{41}$ T. Geralis, ${ }^{41}$ V. A. Giakoumopoulou, ${ }^{41}$ A. Kyriakis, ${ }^{41}$ D. Loukas, ${ }^{41}$ A. Psallidas, ${ }^{41}$ I. Topsis-Giotis ${ }^{41}$ A. Agapitos ${ }^{42}$ S. Kesisoglou, ${ }^{42}$ A. Panagiotou, ${ }^{42}$ N. Saoulidou, ${ }^{42}$ E. Tziaferi, ${ }^{42}$ I. Evangelou, ${ }^{43}$ G. Flouris, ${ }^{43}$ C. Foudas ${ }^{43}$ P. Kokkas, ${ }^{43}$ N. Loukas,${ }^{43}$ N. Manthos,${ }^{43}$ I. Papadopoulos,${ }^{43}$ E. Paradas, ${ }^{43}$ J. Strologas,${ }^{43}$ G. Bencze,${ }^{44}$ C. Hajdu,${ }^{44}$ A. Hazi,${ }^{44}$ P. Hidas, ${ }^{44}$ D. Horvath, ${ }^{44, u}$ F. Sikler, ${ }^{44}$ V. Veszpremi, ${ }^{44}$ G. Vesztergombi, ${ }^{44, v}$ A. J. Zsigmond, ${ }^{44}$ N. Beni, ${ }^{45}$ S. Czellar, ${ }^{45}$ J. Karancsi, ${ }^{45, w}$ J. Molnar, ${ }^{45}$ Z. Szillasi, ${ }^{45, c}$ M. Bartók, ${ }^{46, x}$ A. Makovec, ${ }^{46}$ P. Raics, ${ }^{46}$ Z. L. Trocsanyi, ${ }^{46}$ B. Ujvari, ${ }^{46}$ S. Choudhury, ${ }^{47, y}$ P. Mal,${ }^{47}$ K. Mandal,${ }^{47}$ D. K. Sahoo, ${ }^{47}$ N. Sahoo, ${ }^{47}$ S. K. Swain,${ }^{47}$ S. Bansal, ${ }^{48}$ S. B. Beri,${ }^{48}$ V. Bhatnagar,${ }^{48}$ R. Chawla, ${ }^{48}$ R. Gupta, ${ }^{48}$ U. Bhawandeep, ${ }^{48}$ A. K. Kalsi ${ }^{48}$ A. Kaur, ${ }^{48}$ M. Kaur, ${ }^{48}$ R. Kumar, ${ }^{48}$ A. Mehta, ${ }^{48}$ M. Mittal, ${ }^{48}$ J. B. Singh, ${ }^{48}$ G. Walia, ${ }^{48}$ Ashok Kumar, ${ }^{49}$ A. Bhardwaj, ${ }^{49}$ B. C. Choudhary, ${ }^{49}$ R. B. Garg, ${ }^{49}$ S. Malhotra, ${ }^{49}$ M. Naimuddin, ${ }^{49}$ N. Nishu, ${ }^{49}$ K. Ranjan, ${ }^{49}$ R. Sharma, ${ }^{49}$ V. Sharma,${ }^{49}$ S. Bhattacharya,${ }^{50}$ K. Chatterjee,${ }^{50}$ S. Dey,${ }^{50}$ S. Dutta,${ }^{50}$ N. Majumdar,${ }^{50}$ A. Modak, ${ }^{50}$ K. Mondal,${ }^{50}$ S. Mukhopadhyay, ${ }^{50}$ A. Roy, ${ }^{50}$ D. Roy, ${ }^{50}$ S. Roy Chowdhury, ${ }^{50}$ S. Sarkar ${ }^{50}$ M. Sharan, ${ }^{50}$

A. Abdulsalam, ${ }^{51}$ R. Chudasama, ${ }^{51}$ D. Dutta, ${ }^{51}$ V. Jha, ${ }^{51}$ V. Kumar, ${ }^{51}$ A. K. Mohanty, ${ }^{51, c}$ L. M. Pant,${ }^{51}$ P. Shukla, ${ }^{51}$

A. Topkar, ${ }^{51}$ T. Aziz, ${ }^{52}$ S. Banerjee, ${ }^{52}$ S. Bhowmik, ${ }^{52, \mathrm{z}}$ R. M. Chatterjee, ${ }^{52}$ R. K. Dewanjee, ${ }^{52}$ S. Dugad, ${ }^{52}$ S. Ganguly, ${ }^{52}$ S. Ghosh, ${ }^{52}$ M. Guchait, ${ }^{52}$ A. Gurtu, ${ }^{52, a a}$ Sa. Jain, ${ }^{52}$ G. Kole, ${ }^{52}$ S. Kumar, ${ }^{52}$ B. Mahakud, ${ }^{52}$ M. Maity, ${ }^{52, z}$ G. Majumder, ${ }^{52}$ K. Mazumdar, ${ }^{52}$ S. Mitra, ${ }^{52}$ G. B. Mohanty, ${ }^{52}$ B. Parida,${ }^{52}$ T. Sarkar, ${ }^{52, z}$ N. Sur, ${ }^{52}$ B. Sutar, ${ }^{52}$ N. Wickramage, ${ }^{52, b b}$ S. Chauhan, ${ }^{53}$ S. Dube ${ }^{53}$ A. Kapoor, ${ }^{53}$ K. Kothekar, ${ }^{53}$ S. Sharma, ${ }^{53}$ H. Bakhshiansohi, ${ }^{54}$ H. Behnamian, ${ }^{54}$ S. M. Etesami, ${ }^{54, \mathrm{cc}}$ A. Fahim, ${ }^{54, \mathrm{dd}}$ M. Khakzad, ${ }^{54}$ M. Mohammadi Najafabadi, ${ }^{54}$ M. Naseri, ${ }^{54}$ S. Paktinat Mehdiabadi,${ }^{54}$ F. Rezaei Hosseinabadi, ${ }^{54}$ B. Safarzadeh,${ }^{54, \text { ee }}$ M. Zeinali, ${ }^{54}$ M. Felcini, ${ }^{55}$ M. Grunewald,${ }^{55}$ M. Abbrescia,${ }^{56 a, 56 b}$ C. Calabria,${ }^{56,56 \mathrm{~b}}$ C. Caputo, ${ }^{56 \mathrm{a}, 56 \mathrm{~b}}$ A. Colaleo, ${ }^{56 \mathrm{a}}$ D. Creanza, ${ }^{56 \mathrm{a}, 56 \mathrm{c}}$ L. Cristella ${ }^{56 \mathrm{a}, 56 \mathrm{~b}}$ N. De Filippis,${ }^{56,56 \mathrm{c}}$ M. De Palma, ${ }^{56 a, 56 b}$ L. Fiore, ${ }^{56 \mathrm{a}}$ G. Iaselli, ${ }^{56 a, 56 \mathrm{c}}$ G. Maggi, ${ }^{56 a, 56 \mathrm{c}}$ M. Maggi, ${ }^{56 \mathrm{a}}$ G. Miniello, ${ }^{56 a, 56 \mathrm{~b}} \mathrm{~S}$. My, ${ }^{56 a, 56 \mathrm{c}}$ S. Nuzzo, ${ }^{56 a, 56 \mathrm{~b}}$ A. Pompili, ${ }^{56 a, 56 b}$ G. Pugliese, ${ }^{56 a, 56 c}$ R. Radogna,${ }^{56 a, 56 b}$ A. Ranieri, ${ }^{56 a}$ G. Selvaggi, ${ }^{56 a, 56 b}$ L. Silvestris, ${ }^{56 a, c}$ R. Venditti, ${ }^{56 a, 56 b}$ G. Abbiendi, ${ }^{57 \mathrm{a}}$ C. Battilana, ${ }^{57 \mathrm{a}, \mathrm{c}}$ A. C. Benvenuti, ${ }^{57 \mathrm{a}}$ D. Bonacorsi, ${ }^{57 \mathrm{a}, 57 \mathrm{~b}}$ S. Braibant-Giacomelli, ${ }^{57 \mathrm{a}, 57 \mathrm{~b}}$ L. Brigliadori, ${ }^{57 \mathrm{a}, 57 \mathrm{~b}}$ R. Campanini, ${ }^{57,57 b}$ P. Capiluppi, ${ }^{57 a, 57 b}$ A. Castro, ${ }^{57,57 b}$ F. R. Cavallo, ${ }^{57 a}$ S. S. Chhibra, ${ }^{57,57 b}$ G. Codispoti, ${ }^{57 a, 57 b}$ M. Cuffiani, ${ }^{57 a, 57 b}$ G. M. Dallavalle, ${ }^{57 a}$ F. Fabbri, ${ }^{57 a}$ A. Fanfani, ${ }^{57 a, 57 b}$ D. Fasanella, ${ }^{57 a, 57 b}$ P. Giacomelli, ${ }^{57 a}$ C. Grandi, ${ }^{57 a}$ L. Guiducci, ${ }^{57 a, 57 b}$ S. Marcellini, ${ }^{57 a}$ G. Masetti, ${ }^{57 a}$ A. Montanari, ${ }^{57 a}$ F. L. Navarria, ${ }^{57 a, 57 b}$ A. Perrotta, ${ }^{57 a}$ A. M. Rossi, ${ }^{57 a, 57 b}$ T. Rovelli, ${ }^{57 a, 57 b}$ G. P. Siroli, ${ }^{57 a, 57 b}$ N. Tosi, ${ }^{57 a, 57 b, c}$ R. Travaglini, ${ }^{57 a, 57 b}$ G. Cappello, ${ }^{58 a}$ M. Chiorboli, ${ }^{58 a, 58 b}$ S. Costa, ${ }^{58 a, 58 b}$ A. Di Mattia ${ }^{58 \mathrm{a}}$ F. Giordano,${ }^{58 a, 58 b}$ R. Potenza,${ }^{58,58 b}$ A. Tricomi, ${ }^{58 a, 58 b}$ C. Tuve, ${ }^{58 a, 58 b}$ G. Barbagli, ${ }^{59 a}$ V. Ciulli, ${ }^{59 a, 59 b}$ C. Civinini, ${ }^{59 a}$ R. D'Alessandro, ${ }^{59 a, 59 b}$ E. Focardi, ${ }^{59 a, 59 b}$ V. Gori, ${ }^{59 a, 59 b}$ P. Lenzi, ${ }^{59 a, 59 b}$ M. Meschini, ${ }^{59 a}$ S. Paoletti, ${ }^{59 a}$ G. Sguazzoni,${ }^{59 a}$ L. Viliani, ${ }^{59 a, 59 b, c}$ L. Benussi,${ }^{60}$ S. Bianco,${ }^{60}$ F. Fabbri, ${ }^{60}$ D. Piccolo, ${ }^{60}$ F. Primavera,${ }^{60, c}$ V. Calvelli, ${ }^{61 a, 61 b}$ F. Ferro, ${ }^{61 \mathrm{a}}$ M. Lo Vetere, ${ }^{61 \mathrm{a}, 61 \mathrm{~b}}$ M. R. Monge, ${ }^{61 \mathrm{a}, 61 \mathrm{~b}}$ E. Robutti, ${ }^{61 \mathrm{a}}$ S. Tosi, ${ }^{61 \mathrm{a}, 61 \mathrm{~b}}$ L. Brianza, ${ }^{62 \mathrm{a}}$ M. E. Dinardo, ${ }^{62 \mathrm{a}, 62 \mathrm{~b}}$ S. Fiorendi, ${ }^{62 a, 62 b}$ S. Gennai, ${ }^{62 a}$ R. Gerosa, ${ }^{62 a, 62 b}$ A. Ghezzi, ${ }^{62 a, 62 b}$ P. Govoni, ${ }^{62 a, 62 b}$ S. Malvezzi, ${ }^{62 a}$ R. A. Manzoni, ${ }^{62 a, 62 b, c}$ B. Marzocchi, ${ }^{62 a, 62 b}$ D. Menasce, ${ }^{62 a}$ L. Moroni, ${ }^{62 a}$ M. Paganoni, ${ }^{62 a, 62 b}$ D. Pedrini, ${ }^{62 a}$ S. Ragazzi, ${ }^{62 a, 62 b}$ N. Redaelli, ${ }^{62 a}$ T. Tabarelli de Fatis, ${ }^{62 a, 62 b}$ S. Buontempo, ${ }^{63 a}$ N. Cavallo, ${ }^{63 a, 63 c}$ S. Di Guida, ${ }^{63 a, 63 d, c}$ M. Esposito, ${ }^{63 a, 63 b}$ F. Fabozzi, ${ }^{63 a, 63 c}$ A. O. M. Iorio, ${ }^{63 a, 63 b}$ G. Lanza, ${ }^{63 a}$ L. Lista, ${ }^{63 a}$ S. Meola, ${ }^{63 a, 63 d, c}$ M. Merola, ${ }^{63 a}$ P. Paolucci, ${ }^{63 a, c}$ C. Sciacca, ${ }^{63 a, 63 b}$ F. Thyssen, ${ }^{63 a}$

P. Azzi,${ }^{64 a, c}$ N. Bacchetta, ${ }^{64 a}$ L. Benato,${ }^{64 a, 64 b}$ D. Bisello, ${ }^{64 a, 64 b}$ A. Boletti, ${ }^{64 a, 64 b}$ R. Carlin,,${ }^{64 a, 64 b}$ P. Checchia, ${ }^{64 a}$ M. Dall'Osso, ${ }^{64 a, 64 b, c}$ T. Dorigo, ${ }^{64 a}$ U. Dosselli, ${ }^{64 a}$ F. Gasparini, ${ }^{64 a, 64 b}$ U. Gasparini, ${ }^{64 a, 64 b}$ A. Gozzelino, ${ }^{64 a}$ S. Lacaprara, ${ }^{64 a}$ M. Margoni, ${ }^{64 a, 64 b}$ G. Maron, ${ }^{64 a, f f}$ A. T. Meneguzzo ${ }^{64 a, 64 b}$ F. Montecassiano, ${ }^{64 a}$ M. Passaseo, ${ }^{64 a}$ J. Pazzini, ${ }^{64 a, 64 b, c}$ N. Pozzobon, ${ }^{64 a, 64 b}$ P. Ronchese ${ }^{64 a, 64 b}$ F. Simonetto, ${ }^{64 a, 64 b}$ E. Torassa, ${ }^{64 a}$ M. Tosi,${ }^{64 a, 64 b}$ M. Zanetti, ${ }^{64 a}$ P. Zotto, ${ }^{64 a, 64 b}$ A. Zucchetta, ${ }^{64 a, 64 b, c}$ G. Zumerle, ${ }^{64 a, 64 b}$ A. Braghieri, ${ }^{65 a}$ A. Magnani, ${ }^{65 a, 65 b}$ P. Montagna, ${ }^{65 a, 65 b}$ S. P. Ratti, ${ }^{65 a, 65 b}$ V. Re, ${ }^{65 a}$ 
C. Riccardi, ${ }^{65 a, 65 b}$ P. Salvini, ${ }^{65 a}$ I. Vai, ${ }^{65 a, 65 b}$ P. Vitulo, ${ }^{65 a, 65 b}$ L. Alunni Solestizi, ${ }^{66 a, 66 b}$ G. M. Bilei, ${ }^{66 a}$ D. Ciangottini ${ }^{66 a, 66 b, c}$

L. Fanò ${ }^{66 a, 66 b}$ P. Lariccia, ${ }^{66 a, 66 b}$ G. Mantovani, ${ }^{66 a, 66 b}$ M. Menichelli, ${ }^{66 a}$ A. Saha ${ }^{66 a}$ A. Santocchia ${ }^{66 a, 66 b}$ K. Androsov, ${ }^{67 a, g g}$ P. Azzurri, ${ }^{67 a, c}$ G. Bagliesi, ${ }^{67 a}$ J. Bernardini, ${ }^{67 a}$ T. Boccali, ${ }^{67 a}$ R. Castaldi, ${ }^{67 a}$ M. A. Ciocci ${ }^{67 a, g g}$ R. Dell'Orso, ${ }^{67 a}$

S. Donato, ${ }^{67 a, 67 c, c}$ G. Fedi, ${ }^{67 a}$ L. Foà,${ }^{67 a, 67 c, a}$ A. Giassi ${ }^{67 a}$ M. T. Grippo, ${ }^{67 a, g g}$ F. Ligabue, ${ }^{67 a, 67 c}$ T. Lomtadze, ${ }^{67 a}$ L. Martini, ${ }^{67 a, 67 b}$ A. Messineo, ${ }^{67 a, 67 b}$ F. Palla, ${ }^{67 a}$ A. Rizzi, ${ }^{67 a, 67 b}$ A. Savoy-Navarro, ${ }^{67 a, h h}$ A. T. Serban, ${ }^{67 a}$ P. Spagnolo, ${ }^{67 a}$ R. Tenchini, ${ }^{67 \mathrm{a}}$ G. Tonelli, ${ }^{67 \mathrm{a}, 67 \mathrm{~b}}$ A. Venturi, ${ }^{67 \mathrm{a}}$ P. G. Verdini, ${ }^{67 \mathrm{a}}$ L. Barone, ${ }^{68 \mathrm{a}, 68 \mathrm{~b}}$ F. Cavallari, ${ }^{68 \mathrm{a}}$ G. D'imperio, ${ }^{68 \mathrm{a}, 68 \mathrm{~b}, \mathrm{c}}$ D. Del Re,${ }^{68 a, 68 b, c}$ M. Diemoz, ${ }^{68 a}$ S. Gelli, ${ }^{68 a, 68 b}$ C. Jorda,${ }^{68 a}$ E. Longo ${ }^{68 a, 68 b}$ F. Margaroli, ${ }^{68 a, 68 b}$ P. Meridiani,${ }^{68 a}$

G. Organtini, ${ }^{68 a, 68 b}$ R. Paramatti ${ }^{68 a}$ F. Preiato, ${ }^{68 a, 68 b}$ S. Rahatlou, ${ }^{68 a, 68 b}$ C. Rovelli, ${ }^{68 a}$ F. Santanastasio, ${ }^{68 a, 68 b}$

P. Traczyk,${ }^{68 a, 68 b, c}$ N. Amapane,${ }^{69 a, 69 b}$ R. Arcidiacono, ${ }^{69 a, 69 c, c}$ S. Argiro, ${ }^{69 a, 69 b}$ M. Arneodo,,${ }^{69 a, 69 c}$ R. Bellan, ${ }^{69 a, 69 b}$ C. Biino,${ }^{69 a}$ N. Cartiglia, ${ }^{69 a}$ M. Costa ${ }^{69 a, 69 b}$ R. Covarelli ${ }^{69 a, 69 b}$ A. Degano, ${ }^{69 a, 69 b}$ N. Demaria, ${ }^{69 a}$ L. Finco, ${ }^{69 a, 69 b, c}$ B. Kiani, ${ }^{69 a, 69 b}$ C. Mariotti ${ }^{69 a}$ S. Maselli, ${ }^{69 a}$ E. Migliore ${ }^{69 a, 69 b}$ V. Monaco ${ }^{69 a, 69 b}$ E. Monteil, ${ }^{69 a, 69 b}$ M. M. Obertino, ${ }^{69 a, 69 b}$ L. Pacher ${ }^{69 a, 69 b}$ N. Pastrone ${ }^{69 \mathrm{a}}$ M. Pelliccioni ${ }^{69 \mathrm{a}}$ G. L. Pinna Angioni, ${ }^{69 \mathrm{a}, 69 \mathrm{~b}}$ F. Ravera,${ }^{69 a, 69 \mathrm{~b}}$ A. Romero, ${ }^{69 a, 69 \mathrm{~b}}$ M. Ruspa,${ }^{69 a, 69 \mathrm{c}}$ R. Sacchi ${ }^{69 a, 69 b}$ A. Solano,${ }^{69 a, 69 b}$ A. Staiano ${ }^{69 a}$ S. Belforte, ${ }^{70 a}$ V. Candelise,${ }^{70 a, 70 b}$ M. Casarsa, ${ }^{70 a}$ F. Cossutti, ${ }^{70 a}$ G. Della Ricca, ${ }^{70 a, 70 b}$ B. Gobbo, ${ }^{70 a}$ C. La Licata, ${ }^{70 a, 70 b}$ M. Marone, ${ }^{70 a, 70 b}$ A. Schizzi, ${ }^{70 a, 70 b}$ A. Zanetti, ${ }^{70 a}$ A. Kropivnitskaya, ${ }^{71}$ S. K. Nam, ${ }^{71}$ D. H. Kim, ${ }^{72}$ G. N. Kim, ${ }^{72}$ M. S. Kim, ${ }^{72}$ D. J. Kong, ${ }^{72}$ S. Lee, ${ }^{72}$ Y. D. Oh, ${ }^{72}$ A. Sakharov, ${ }^{72}$ D. C. Son, ${ }^{72}$ J. A. Brochero Cifuentes, ${ }^{73}$ H. Kim,${ }^{73}$ T. J. Kim ${ }^{73}$ S. Song ${ }^{74}$ S. Choi,${ }^{75}$ Y. Go, ${ }^{75}$ D. Gyun, ${ }^{75}$ B. Hong,${ }^{75}$ H. Kim, ${ }^{75}$ Y. Kim,${ }^{75}$ B. Lee, ${ }^{75}$ K. Lee, ${ }^{75}$ K. S. Lee, ${ }^{75}$ S. Lee, ${ }^{75}$ S. K. Park, ${ }^{75}$ Y. Roh, ${ }^{75}$ H. D. Yoo, ${ }^{76}$ M. Choi, ${ }^{77}$ H. Kim, ${ }^{77}$ J. H. Kim, ${ }^{77}$ J. S. H. Lee ${ }^{77}$ I. C. Park, ${ }^{77}$ G. Ryu ${ }^{77}$ M. S. Ryu, ${ }^{77}$ Y. Choi,${ }^{78}$ J. Goh, ${ }^{78}$ D. Kim,${ }^{78}$ E. Kwon, ${ }^{78}$ J. Lee, ${ }^{78}$ I. Yu, ${ }^{78}$ V. Dudenas,${ }^{79}$ A. Juodagalvis, ${ }^{79}$ J. Vaitkus, ${ }^{79}$ I. Ahmed, ${ }^{80}$ Z. A. Ibrahim, ${ }^{80}$ J. R. Komaragiri, ${ }^{80}$ M. A. B. Md Ali, ${ }^{80, i i}$ F. Mohamad Idris, ${ }^{80, j j}$ W. A. T. Wan Abdullah, ${ }^{80}$ M. N. Yusli, ${ }^{80}$ E. Casimiro Linares ${ }^{81}$ H. Castilla-Valdez ${ }^{81}$ E. De La Cruz-Burelo, ${ }^{81}$ I. Heredia-De La Cruz, ${ }^{81, k k}$ A. Hernandez-Almada, ${ }^{81}$ R. Lopez-Fernandez, ${ }^{81}$ A. Sanchez-Hernandez, ${ }^{81}$

S. Carrillo Moreno, ${ }^{82}$ F. Vazquez Valencia, ${ }^{82}$ I. Pedraza, ${ }^{83}$ H. A. Salazar Ibarguen, ${ }^{83}$ A. Morelos Pineda, ${ }^{84}$ D. Krofcheck ${ }^{85}$ P. H. Butler, ${ }^{86}$ A. Ahmad, ${ }^{87}$ M. Ahmad ${ }^{87}$ Q. Hassan, ${ }^{87}$ H. R. Hoorani, ${ }^{87}$ W. A. Khan, ${ }^{87}$ T. Khurshid ${ }^{87}$ M. Shoaib, ${ }^{87}$ H. Bialkowska, ${ }^{88}$ M. Bluj, ${ }^{88}$ B. Boimska, ${ }^{88}$ T. Frueboes,${ }^{88}$ M. Górski, ${ }^{88}$ M. Kazana, ${ }^{88}$ K. Nawrocki, ${ }^{88}$

K. Romanowska-Rybinska, ${ }^{88}$ M. Szleper ${ }^{88}$ P. Zalewski, ${ }^{88}$ G. Brona, ${ }^{89}$ K. Bunkowski, ${ }^{89}$ A. Byszuk,${ }^{89,11}$ K. Doroba, ${ }^{89}$ A. Kalinowski ${ }^{89}$ M. Konecki, ${ }^{89}$ J. Krolikowski, ${ }^{89}$ M. Misiura, ${ }^{89}$ M. Olszewski, ${ }^{89}$ M. Walczak, ${ }^{89}$ P. Bargassa, ${ }^{90}$

C. Beirão Da Cruz E Silva, ${ }^{90}$ A. Di Francesco, ${ }^{90}$ P. Faccioli, ${ }^{90}$ P. G. Ferreira Parracho, ${ }^{90}$ M. Gallinaro, ${ }^{90}$ J. Hollar,${ }^{90}$

N. Leonardo, ${ }^{90}$ L. Lloret Iglesias, ${ }^{90}$ F. Nguyen, ${ }^{90}$ J. Rodrigues Antunes, ${ }^{90}$ J. Seixas,${ }^{90}$ O. Toldaiev, ${ }^{90}$ D. Vadruccio,${ }^{90}$

J. Varela, ${ }^{90}$ P. Vischia, ${ }^{90}$ S. Afanasiev,${ }^{91}$ P. Bunin,${ }^{91}$ M. Gavrilenko, ${ }^{91}$ I. Golutvin, ${ }^{91}$ I. Gorbunov, ${ }^{91}$ A. Kamenev, ${ }^{91}$ V. Karjavin, ${ }^{91}$ A. Lanev, ${ }^{91}$ A. Malakhov, ${ }^{91}$ V. Matveev,${ }^{91, m m, n n}$ P. Moisenz, ${ }^{91}$ V. Palichik, ${ }^{91}$ V. Perelygin, ${ }^{91}$ S. Shmatov,${ }^{91}$ S. Shulha,${ }^{91}$ N. Skatchkov, ${ }^{91}$ V. Smirnov, ${ }^{91}$ A. Zarubin, ${ }^{91}$ V. Golovtsov, ${ }^{92}$ Y. Ivanov, ${ }^{92}$ V. Kim,${ }^{92,00}$ E. Kuznetsova, ${ }^{92}$ P. Levchenko, ${ }^{92}$ V. Murzin, ${ }^{92}$ V. Oreshkin, ${ }^{92}$ I. Smirnov, ${ }^{92}$ V. Sulimov, ${ }^{92}$ L. Uvarov, ${ }^{92}$ S. Vavilov, ${ }^{92}$ A. Vorobyev, ${ }^{92}$ Yu. Andreev, ${ }^{93}$ A. Dermenev, ${ }^{93}$ S. Gninenko, ${ }^{93}$ N. Golubev, ${ }^{93}$ A. Karneyeu, ${ }^{93}$ M. Kirsanov ${ }^{93}$ N. Krasnikov,${ }^{93}$ A. Pashenkov, ${ }^{93}$ D. Tlisov, ${ }^{93}$ A. Toropin, ${ }^{93}$ V. Epshteyn, ${ }^{94}$ V. Gavrilov,${ }^{94}$ N. Lychkovskaya, ${ }^{94}$ V. Popov, ${ }^{94}$ I. Pozdnyakov,${ }^{94}$ G. Safronov, ${ }^{94}$ A. Spiridonov, ${ }^{94}$ E. Vlasov, ${ }^{94}$ A. Zhokin, ${ }^{94}$ A. Bylinkin, ${ }^{95}$ V. Andreev, ${ }^{96}$ M. Azarkin,,${ }^{96, n n}$ I. Dremin, ${ }^{96, n n}$ M. Kirakosyan, ${ }^{96}$ A. Leonidov, ${ }^{96, n n}$ G. Mesyats, ${ }^{96}$ S. V. Rusakov, ${ }^{96}$ A. Baskakov, ${ }^{97}$ A. Belyaev, ${ }^{97}$ E. Boos,${ }^{97}$ M. Dubinin,,${ }^{97, p p}$ L. Dudko, ${ }^{97}$ A. Ershov, ${ }^{97}$ A. Gribushin, ${ }^{97}$ V. Klyukhin, ${ }^{97}$ O. Kodolova, ${ }^{97}$ I. Lokhtin, ${ }^{97}$ I. Myagkov, ${ }^{97}$ S. Obraztsov, ${ }^{97}$

S. Petrushanko, ${ }^{97}$ V. Savrin, ${ }^{97}$ A. Snigirev, ${ }^{97}$ I. Azhgirey, ${ }^{98}$ I. Bayshev,${ }^{98}$ S. Bitioukov, ${ }^{98}$ V. Kachanov,${ }^{98}$ A. Kalinin, ${ }^{98}$ D. Konstantinov, ${ }^{98}$ V. Krychkine, ${ }^{98}$ V. Petrov, ${ }^{98}$ R. Ryutin, ${ }^{98}$ A. Sobol,${ }^{98}$ L. Tourtchanovitch, ${ }^{98}$ S. Troshin, ${ }^{98}$ N. Tyurin, ${ }^{98}$ A. Uzunian, ${ }^{98}$ A. Volkov, ${ }^{98}$ P. Adzic, ${ }^{99,4 q}$ P. Cirkovic, ${ }^{99}$ J. Milosevic, ${ }^{99}$ V. Rekovic, ${ }^{99}$ J. Alcaraz Maestre, ${ }^{100}$ E. Calvo, ${ }^{100}$ M. Cerrada, ${ }^{100}$ M. Chamizo Llatas, ${ }^{100}$ N. Colino, ${ }^{100}$ B. De La Cruz, ${ }^{100}$ A. Delgado Peris, ${ }^{100}$ A. Escalante Del Valle, ${ }^{100}$ C. Fernandez Bedoya, ${ }^{100}$ J. P. Fernández Ramos, ${ }^{100}$ J. Flix, ${ }^{100}$ M. C. Fouz,${ }^{100}$ P. Garcia-Abia, ${ }^{100}$ O. Gonzalez Lopez, ${ }^{100}$ S. Goy Lopez,${ }^{100}$ J. M. Hernandez,${ }^{100}$ M. I. Josa ${ }^{100}$ E. Navarro De Martino, ${ }^{100}$ A. Pérez-Calero Yzquierdo, ${ }^{100}$

J. Puerta Pelayo, ${ }^{100}$ A. Quintario Olmeda,${ }^{100}$ I. Redondo,${ }^{100}$ L. Romero, ${ }^{100}$ J. Santaolalla, ${ }^{100}$ M. S. Soares ${ }^{100}$ C. Albajar, ${ }^{101}$ J. F. de Trocóniz, ${ }^{101}$ M. Missiroli, ${ }^{101}$ D. Moran, ${ }^{101}$ J. Cuevas, ${ }^{102}$ J. Fernandez Menendez ${ }^{102}$ S. Folgueras,${ }^{102}$ I. Gonzalez Caballero, ${ }^{102}$ E. Palencia Cortezon, ${ }^{102}$ J. M. Vizan Garcia, ${ }^{102}$ I. J. Cabrillo, ${ }^{103}$ A. Calderon, ${ }^{103}$ J. R. Castiñeiras De Saa, ${ }^{103}$ P. De Castro Manzano, ${ }^{103}$ M. Fernandez, ${ }^{103}$ J. Garcia-Ferrero, ${ }^{103}$ G. Gomez, ${ }^{103}$ A. Lopez Virto, ${ }^{103}$ J. Marco ${ }^{103}$ R. Marco, ${ }^{103}$ C. Martinez Rivero, ${ }^{103}$ F. Matorras,${ }^{103}$ J. Piedra Gomez, ${ }^{103}$ T. Rodrigo, ${ }^{103}$ A. Y. Rodríguez-Marrero, ${ }^{103}$ A. Ruiz-Jimeno, ${ }^{103}$ L. Scodellaro, ${ }^{103}$ N. Trevisani, ${ }^{103}$ I. Vila, ${ }^{103}$ R. Vilar Cortabitarte, ${ }^{103}$ 
D. Abbaneo, ${ }^{104}$ E. Auffray, ${ }^{104}$ G. Auzinger, ${ }^{104}$ M. Bachtis, ${ }^{104}$ P. Baillon, ${ }^{104}$ A. H. Ball, ${ }^{104}$ D. Barney, ${ }^{104}$ A. Benaglia, ${ }^{104}$ J. Bendavid, ${ }^{104}$ L. Benhabib, ${ }^{104}$ G. M. Berruti, ${ }^{104}$ P. Bloch,${ }^{104}$ A. Bocci, ${ }^{104}$ A. Bonato, ${ }^{104}$ C. Botta, ${ }^{104}$ H. Breuker,${ }^{104}$ T. Camporesi, ${ }^{104}$ R. Castello, ${ }^{104}$ G. Cerminara ${ }^{104}$ M. D'Alfonso, ${ }^{104}$ D. d'Enterria, ${ }^{104}$ A. Dabrowski,${ }^{104}$ V. Daponte, ${ }^{104}$ A. David, ${ }^{104}$ M. De Gruttola, ${ }^{104}$ F. De Guio, ${ }^{104}$ A. De Roeck, ${ }^{104}$ S. De Visscher, ${ }^{104}$ E. Di Marco, ${ }^{104, \text { rr }}$ M. Dobson, ${ }^{104}$ M. Dordevic, ${ }^{104}$ B. Dorney, ${ }^{104}$ T. du Pree, ${ }^{104}$ D. Duggan, ${ }^{104}$ M. Dünser, ${ }^{104}$ N. Dupont, ${ }^{104}$ A. Elliott-Peisert, ${ }^{104}$ G. Franzoni, ${ }^{104}$ J. Fulcher, ${ }^{104}$ W. Funk, ${ }^{104}$ D. Gigi, ${ }^{104}$ K. Gill, ${ }^{104}$ D. Giordano,${ }^{104}$ M. Girone,${ }^{104}$ F. Glege,${ }^{104}$ R. Guida, ${ }^{104}$ S. Gundacker, ${ }^{104}$ M. Guthoff, ${ }^{104}$ J. Hammer, ${ }^{104}$ P. Harris, ${ }^{104}$ J. Hegeman, ${ }^{104}$ V. Innocente, ${ }^{104}$ P. Janot, ${ }^{104}$ H. Kirschenmann, ${ }^{104}$ M. J. Kortelainen, ${ }^{104}$ K. Kousouris, ${ }^{104}$ K. Krajczar, ${ }^{104}$ P. Lecoq, ${ }^{104}$ C. Lourenço, ${ }^{104}$ M. T. Lucchini, ${ }^{104}$ N. Magini, ${ }^{104}$ L. Malgeri, ${ }^{104}$ M. Mannelli, ${ }^{104}$ A. Martelli, ${ }^{104}$ L. Masetti, ${ }^{104}$ F. Meijers, ${ }^{104}$ S. Mersi, ${ }^{104}$ E. Meschi, ${ }^{104}$ F. Moortgat,${ }^{104}$ S. Morovic, ${ }^{104}$ M. Mulders, ${ }^{104}$ M. V. Nemallapudi, ${ }^{104}$ H. Neugebauer, ${ }^{104}$ S. Orfanelli, ${ }^{104, s s}$ L. Orsini, ${ }^{104}$ L. Pape,${ }^{104}$ E. Perez, ${ }^{104}$ M. Peruzzi, ${ }^{104}$ A. Petrilli, ${ }^{104}$ G. Petrucciani, ${ }^{104}$ A. Pfeiffer, ${ }^{104}$ M. Pierini, ${ }^{104}$ D. Piparo, ${ }^{104}$ A. Racz, ${ }^{104}$ T. Reis, ${ }^{104}$ G. Rolandi, ${ }^{104, t t}$ M. Rovere, ${ }^{104}$ M. Ruan, ${ }^{104}$ H. Sakulin, ${ }^{104}$ C. Schäfer,${ }^{104}$ C. Schwick,${ }^{104}$ M. Seidel, ${ }^{104}$ A. Sharma,${ }^{104}$ P. Silva, ${ }^{104}$ M. Simon, ${ }^{104}$ P. Sphicas, ${ }^{104, \text { uu }}$ J. Steggemann, ${ }^{104}$ B. Stieger,${ }^{104}$ M. Stoye,${ }^{104}$ Y. Takahashi, ${ }^{104}$ D. Treille, ${ }^{104}$ A. Triossi, ${ }^{104}$ A. Tsirou, ${ }^{104}$ G. I. Veres, ${ }^{104, v}$ N. Wardle, ${ }^{104}$ H. K. Wöhri, ${ }^{104}$ A. Zagozdzinska, ${ }^{104,11}$ W. D. Zeuner, ${ }^{104}$ W. Bertl, ${ }^{105}$ K. Deiters, ${ }^{105}$ W. Erdmann, ${ }^{105}$ R. Horisberger, ${ }^{105}$ Q. Ingram, ${ }^{105}$ H. C. Kaestli, ${ }^{105}$ D. Kotlinski, ${ }^{105}$ U. Langenegger, ${ }^{105}$ D. Renker, ${ }^{105}$ T. Rohe, ${ }^{105}$ F. Bachmair, ${ }^{106}$ L. Bäni, ${ }^{106}$ L. Bianchini, ${ }^{106}$ B. Casal, ${ }^{106}$ G. Dissertori, ${ }^{106}$ M. Dittmar, ${ }^{106}$ M. Donegà, ${ }^{106}$ P. Eller,${ }^{106}$ C. Grab,${ }^{106}$ C. Heidegger,${ }^{106}$ D. Hits, ${ }^{106}$ J. Hoss, ${ }^{106}$ G. Kasieczka, ${ }^{106}$ P. Lecomte, ${ }^{106, a}$ W. Lustermann, ${ }^{106}$ B. Mangano, ${ }^{106}$ M. Marionneau, ${ }^{106}$ P. Martinez Ruiz del Arbol, ${ }^{106}$ M. Masciovecchio, ${ }^{106}$ D. Meister, ${ }^{106}$ F. Micheli, ${ }^{106}$ P. Musella, ${ }^{106}$ F. Nessi-Tedaldi, ${ }^{106}$ F. Pandolfi, ${ }^{106}$ J. Pata, ${ }^{106}$ F. Pauss, ${ }^{106}$ L. Perrozzi, ${ }^{106}$ M. Quittnat ${ }^{106}$ M. Rossini, ${ }^{106}$ M. Schönenberger, ${ }^{106}$ A. Starodumov, ${ }^{106, v v}$ M. Takahashi, ${ }^{106}$ V. R. Tavolaro, ${ }^{106}$ K. Theofilatos, ${ }^{106}$ R. Wallny, ${ }^{106}$ T. K. Aarrestad, ${ }^{107}$ C. Amsler, ${ }^{107, w w}$ L. Caminada, ${ }^{107}$ M. F. Canelli, ${ }^{107}$ V. Chiochia, ${ }^{107}$ A. De Cosa,${ }^{107}$ C. Galloni, ${ }^{107}$ A. Hinzmann, ${ }^{107}$ T. Hreus ${ }^{107}$ B. Kilminster ${ }^{107}$ C. Lange,${ }^{107}$ J. Ngadiuba,${ }^{107}$ D. Pinna,${ }^{107}$ G. Rauco, ${ }^{107}$ P. Robmann, ${ }^{107}$ F. J. Ronga, ${ }^{107}$ D. Salerno, ${ }^{107}$ Y. Yang, ${ }^{107}$ M. Cardaci, ${ }^{108}$ K. H. Chen, ${ }^{108}$ T. H. Doan, ${ }^{108}$ Sh. Jain, ${ }^{108}$ R. Khurana, ${ }^{108}$ M. Konyushikhin, ${ }^{108}$ C. M. Kuo, ${ }^{108}$ W. Lin, ${ }^{108}$ Y. J. Lu, ${ }^{108}$ A. Pozdnyakov, ${ }^{108}$ S. S. Yu, ${ }^{108}$ Arun Kumar, ${ }^{109}$ P. Chang, ${ }^{109}$ Y. H. Chang, ${ }^{109}$ Y. W. Chang, ${ }^{109}$ Y. Chao, ${ }^{109}$ K. F. Chen, ${ }^{109}$ P. H. Chen, ${ }^{109}$ C. Dietz, ${ }^{109}$ F. Fiori, ${ }^{109}$ U. Grundler, ${ }^{109}$ W.-S. Hou, ${ }^{109}$ Y. Hsiung, ${ }^{109}$ Y. F. Liu, ${ }^{109}$ R.-S. Lu, ${ }^{109}$ M. Miñano Moya, ${ }^{109}$ E. Petrakou, ${ }^{109}$ J. f. Tsai, ${ }^{109}$ Y. M. Tzeng, ${ }^{109}$ B. Asavapibhop, ${ }^{110}$ K. Kovitanggoon, ${ }^{110}$ G. Singh, ${ }^{110}$ N. Srimanobhas, ${ }^{110}$ N. Suwonjandee, ${ }^{110}$ A. Adiguzel, ${ }^{111}$ M. N. Bakirci, ${ }^{111, x x}$ A. Bat, ${ }^{111}$ Z. S. Demiroglu, ${ }^{111}$ C. Dozen, ${ }^{111}$ F. H. Gecit, ${ }^{11}$ S. Girgis, ${ }^{111}$ G. Gokbulut, ${ }^{111}$ Y. Guler, ${ }^{111}$ E. Gurpinar, ${ }^{111}$ I. Hos, ${ }^{111}$ E. E. Kangal, ${ }^{11, y y}$ A. Kayis Topaksu, ${ }^{111}$ G. Onengut, ${ }^{11, z z}$ M. Ozcan, ${ }^{111}$

K. Ozdemir, ${ }^{111, \text { aaa }}$ S. Ozturk, ${ }^{11, \text { xx }}$ D. Sunar Cerci, ${ }^{111, \text { bbb }}$ B. Tali, ${ }^{11, b b b}$ H. Topakli, ${ }^{11, x x}$ C. Zorbilmez,${ }^{111}$ B. Bilin, ${ }^{112}$ S. Bilmis, ${ }^{112}$ B. Isildak, ${ }^{12, \text { ccc }}$ G. Karapinar, ${ }^{112, \text { ddd }}$ M. Yalvac, ${ }^{112}$ M. Zeyrek, ${ }^{112}$ E. Gülmez, ${ }^{113}$ M. Kaya, ${ }^{113, \text { eee }}$ O. Kaya, ${ }^{113, f f f}$ E. A. Yetkin, ${ }^{113, \text { ggg }}$ T. Yetkin, ${ }^{113 \text {,hhh }}$ A. Cakir, ${ }^{114}$ K. Cankocak, ${ }^{114}$ S. Sen, ${ }^{114, \text { iii }}$ F. I. Vardarl1, ${ }^{114}$ B. Grynyov, ${ }^{115}$ L. Levchuk, ${ }^{116}$

P. Sorokin, ${ }^{116}$ R. Aggleton, ${ }^{117}$ F. Ball, ${ }^{117}$ L. Beck, ${ }^{117}$ J. J. Brooke, ${ }^{117}$ E. Clement, ${ }^{117}$ D. Cussans, ${ }^{117}$ H. Flacher,${ }^{117}$

J. Goldstein, ${ }^{117}$ M. Grimes, ${ }^{117}$ G. P. Heath, ${ }^{117}$ H. F. Heath, ${ }^{117}$ J. Jacob, ${ }^{117}$ L. Kreczko, ${ }^{117}$ C. Lucas, ${ }^{117}$ Z. Meng, ${ }^{117}$ D. M. Newbold, ${ }^{117, j j j}$ S. Paramesvaran, ${ }^{117}$ A. Poll, ${ }^{117}$ T. Sakuma, ${ }^{117}$ S. Seif El Nasr-storey, ${ }^{117}$ S. Senkin, ${ }^{117}$ D. Smith, ${ }^{117}$ V. J. Smith, ${ }^{117}$ K. W. Bell, ${ }^{118}$ A. Belyaev, ${ }^{118, k k k}$ C. Brew, ${ }^{118}$ R. M. Brown, ${ }^{118}$ L. Calligaris, ${ }^{118}$ D. Cieri, ${ }^{118}$ D. J. A. Cockerill, ${ }^{118}$ J. A. Coughlan, ${ }^{118}$ K. Harder,${ }^{118}$ S. Harper, ${ }^{118}$ E. Olaiya, ${ }^{118}$ D. Petyt,${ }^{118}$

C. H. Shepherd-Themistocleous, ${ }^{118}$ A. Thea, ${ }^{118}$ I. R. Tomalin, ${ }^{118}$ T. Williams, ${ }^{118}$ S. D. Worm, ${ }^{118}$ M. Baber, ${ }^{119}$ R. Bainbridge, ${ }^{119}$ O. Buchmuller, ${ }^{119}$ A. Bundock, ${ }^{119}$ D. Burton, ${ }^{119}$ S. Casasso, ${ }^{119}$ M. Citron, ${ }^{119}$ D. Colling, ${ }^{119}$ L. Corpe,${ }^{119}$ P. Dauncey, ${ }^{119}$ G. Davies, ${ }^{119}$ A. De Wit, ${ }^{119}$ M. Della Negra,${ }^{119}$ P. Dunne,${ }^{119}$ A. Elwood, ${ }^{119}$ D. Futyan, ${ }^{119}$ G. Hall, ${ }^{119}$ G. Iles, ${ }^{119}$ R. Lane, ${ }^{119}$ R. Lucas, ${ }^{19, j j j}$ L. Lyons, ${ }^{119}$ A.-M. Magnan, ${ }^{119}$ S. Malik, ${ }^{119}$ J. Nash, ${ }^{119}$ A. Nikitenko, ${ }^{119, v v}$ J. Pela, ${ }^{119}$ M. Pesaresi, ${ }^{119}$ K. Petridis, ${ }^{119}$ D. M. Raymond, ${ }^{119}$ A. Richards, ${ }^{119}$ A. Rose,${ }^{119}$ C. Seez, ${ }^{119}$ A. Tapper, ${ }^{119}$ K. Uchida, ${ }^{119}$ M. Vazquez Acosta, ${ }^{19,111}$ T. Virdee, ${ }^{119}$ S. C. Zenz, ${ }^{119}$ J. E. Cole, ${ }^{120}$ P. R. Hobson, ${ }^{120}$ A. Khan, ${ }^{120}$ P. Kyberd, ${ }^{120}$ D. Leggat, ${ }^{120}$ D. Leslie, ${ }^{120}$ I. D. Reid, ${ }^{120}$ P. Symonds, ${ }^{120}$ L. Teodorescu, ${ }^{120}$ M. Turner, ${ }^{120}$ A. Borzou, ${ }^{121}$ K. Call, ${ }^{121}$ J. Dittmann, ${ }^{121}$ K. Hatakeyama ${ }^{121}$ H. Liu ${ }^{121}$ N. Pastika,${ }^{121}$ O. Charaf ${ }^{122}$ S. I. Cooper, ${ }^{122}$ C. Henderson, ${ }^{122}$ P. Rumerio, ${ }^{122}$ D. Arcaro, ${ }^{123}$ A. Avetisyan, ${ }^{123}$ T. Bose,${ }^{123}$ D. Gastler, ${ }^{123}$ D. Rankin, ${ }^{123}$ C. Richardson, ${ }^{123}$ J. Rohlf, ${ }^{123}$ L. Sulak, ${ }^{123}$ D. Zou, ${ }^{123}$ J. Alimena, ${ }^{124}$ E. Berry, ${ }^{124}$ D. Cutts, ${ }^{124}$ A. Ferapontov, ${ }^{124}$ A. Garabedian, ${ }^{124}$ J. Hakala, ${ }^{124}$ U. Heintz, ${ }^{124}$ E. Laird, ${ }^{124}$ G. Landsberg, ${ }^{124}$ Z. Mao, ${ }^{124}$ M. Narain, ${ }^{124}$ S. Piperov, ${ }^{124}$ S. Sagir, ${ }^{124}$ R. Syarif, ${ }^{124}$ R. Breedon, ${ }^{125}$ G. Breto, ${ }^{125}$

M. Calderon De La Barca Sanchez, ${ }^{125}$ S. Chauhan, ${ }^{125}$ M. Chertok, ${ }^{125}$ J. Conway, ${ }^{125}$ R. Conway, ${ }^{125}$ P. T. Cox, ${ }^{125}$ 
R. Erbacher, ${ }^{125}$ G. Funk, ${ }^{125}$ M. Gardner, ${ }^{125}$ W. Ko, ${ }^{125}$ R. Lander, ${ }^{125}$ C. Mclean, ${ }^{125}$ M. Mulhearn, ${ }^{125}$ D. Pellett, ${ }^{125}$ J. Pilot, ${ }^{125}$ F. Ricci-Tam, ${ }^{125}$ S. Shalhout, ${ }^{125}$ J. Smith ${ }^{125}$ M. Squires,${ }^{125}$ D. Stolp, ${ }^{125}$ M. Tripathi, ${ }^{125}$ S. Wilbur, ${ }^{125}$ R. Yohay, ${ }^{125}$ R. Cousins, ${ }^{126}$ P. Everaerts, ${ }^{126}$ A. Florent, ${ }^{126}$ J. Hauser, ${ }^{126}$ M. Ignatenko, ${ }^{126}$ D. Saltzberg, ${ }^{126}$ E. Takasugi, ${ }^{126}$ V. Valuev, ${ }^{126}$ M. Weber, ${ }^{126}$ K. Burt, ${ }^{127}$ R. Clare, ${ }^{127}$ J. Ellison, ${ }^{127}$ J. W. Gary, ${ }^{127}$ G. Hanson, ${ }^{127}$ J. Heilman, ${ }^{127}$ M. Ivova PANEVA, ${ }^{127}$ P. Jandir, ${ }^{127}$ E. Kennedy, ${ }^{127}$ F. Lacroix,${ }^{127}$ O. R. Long, ${ }^{127}$ M. Malberti, ${ }^{127}$ M. Olmedo Negrete, ${ }^{127}$ A. Shrinivas, ${ }^{127}$ H. Wei, ${ }^{127}$ S. Wimpenny, ${ }^{127}$ B. R. Yates, ${ }^{127}$ J. G. Branson, ${ }^{128}$ G. B. Cerati, ${ }^{128}$ S. Cittolin, ${ }^{128}$ R. T. D'Agnolo, ${ }^{128}$ M. Derdzinski, ${ }^{128}$ A. Holzner, ${ }^{128}$ R. Kelley, ${ }^{128}$ D. Klein, ${ }^{128}$ J. Letts, ${ }^{128}$ I. Macneill, ${ }^{128}$ D. Olivito, ${ }^{128}$ S. Padhi, ${ }^{128}$ M. Pieri, ${ }^{128}$ M. Sani, ${ }^{128}$ V. Sharma, ${ }^{128}$ S. Simon, ${ }^{128}$ M. Tadel, ${ }^{128}$ A. Vartak, ${ }^{128}$ S. Wasserbaech, ${ }^{128, m m m}$ C. Welke, ${ }^{128}$ F. Würthwein, ${ }^{128}$ A. Yagil, ${ }^{128}$ G. Zevi Della Porta, ${ }^{128}$ J. Bradmiller-Feld, ${ }^{129}$ C. Campagnari, ${ }^{129}$ A. Dishaw, ${ }^{129}$ V. Dutta, ${ }^{129}$ K. Flowers, ${ }^{129}$

M. Franco Sevilla, ${ }^{129}$ P. Geffert, ${ }^{129}$ C. George, ${ }^{129}$ F. Golf, ${ }^{129}$ L. Gouskos, ${ }^{129}$ J. Gran, ${ }^{129}$ J. Incandela, ${ }^{129}$ N. Mccoll, ${ }^{129}$ S. D. Mullin, ${ }^{129}$ J. Richman, ${ }^{129}$ D. Stuart, ${ }^{129}$ I. Suarez, ${ }^{129}$ C. West,${ }^{129}$ J. Yoo, ${ }^{129}$ D. Anderson, ${ }^{130}$ A. Apresyan, ${ }^{130}$ A. Bornheim, ${ }^{130}$ J. Bunn, ${ }^{130}$ Y. Chen, ${ }^{130}$ J. Duarte, ${ }^{130}$ A. Mott, ${ }^{130}$ H. B. Newman, ${ }^{130}$ C. Pena,${ }^{130}$ M. Spiropulu, ${ }^{130}$ J. R. Vlimant ${ }^{130}$ S. Xie, ${ }^{130}$ R. Y. Zhu ${ }^{130}$ M. B. Andrews, ${ }^{131}$ V. Azzolini, ${ }^{131}$ A. Calamba, ${ }^{131}$ B. Carlson, ${ }^{131}$ T. Ferguson, ${ }^{131}$ M. Paulini, ${ }^{131}$ J. Russ, ${ }^{131}$ M. Sun, ${ }^{131}$ H. Vogel, ${ }^{131}$ I. Vorobiev, ${ }^{131}$ J. P. Cumalat, ${ }^{132}$ W. T. Ford, ${ }^{132}$ A. Gaz, ${ }^{132}$ F. Jensen, ${ }^{132}$ A. Johnson, ${ }^{132}$ M. Krohn, ${ }^{132}$ T. Mulholland, ${ }^{132}$ U. Nauenberg, ${ }^{132}$ K. Stenson, ${ }^{132}$ S. R. Wagner,${ }^{132}$ J. Alexander ${ }^{133}$ A. Chatterjee, ${ }^{133}$ J. Chaves, ${ }^{133}$ J. Chu, ${ }^{133}$ S. Dittmer,${ }^{133}$ N. Eggert, ${ }^{133}$ N. Mirman, ${ }^{133}$ G. Nicolas Kaufman, ${ }^{133}$ J. R. Patterson, ${ }^{133}$ A. Rinkevicius, ${ }^{133}$ A. Ryd, ${ }^{133}$ L. Skinnari, ${ }^{133}$ L. Soffi, ${ }^{133}$ W. Sun, ${ }^{133}$ S. M. Tan, ${ }^{133}$ W. D. Teo, ${ }^{133}$ J. Thom, ${ }^{133}$ J. Thompson, ${ }^{133}$ J. Tucker, ${ }^{133}$ Y. Weng, ${ }^{133}$ P. Wittich, ${ }^{133}$ S. Abdullin, ${ }^{134}$ M. Albrow, ${ }^{134}$ G. Apollinari, ${ }^{134}$ S. Banerjee, ${ }^{134}$ L. A. T. Bauerdick, ${ }^{134}$ A. Beretvas, ${ }^{134}$ J. Berryhill, ${ }^{134}$ P. C. Bhat, ${ }^{134}$ G. Bolla, ${ }^{134}$ K. Burkett, ${ }^{134}$ J. N. Butler, ${ }^{134}$ H. W. K. Cheung, ${ }^{134}$ F. Chlebana, ${ }^{134}$ S. Cihangir, ${ }^{134}$ V. D. Elvira, ${ }^{134}$ I. Fisk, ${ }^{134}$ J. Freeman, ${ }^{134}$ E. Gottschalk, ${ }^{134}$ L. Gray, ${ }^{134}$ D. Green, ${ }^{134}$ S. Grünendahl, ${ }^{134}$ O. Gutsche, ${ }^{134}$ J. Hanlon, ${ }^{134}$ D. Hare,${ }^{134}$ R. M. Harris, ${ }^{134}$ S. Hasegawa, ${ }^{134}$ J. Hirschauer, ${ }^{134}$ Z. Hu, ${ }^{134}$ B. Jayatilaka, ${ }^{134}$ S. Jindariani,${ }^{134}$ M. Johnson, ${ }^{134}$ U. Joshi, ${ }^{134}$ B. Klima, ${ }^{134}$ B. Kreis, ${ }^{134}$ S. Lammel,${ }^{134}$ J. Linacre, ${ }^{134}$ D. Lincoln, ${ }^{134}$ R. Lipton, ${ }^{134}$ T. Liu,${ }^{134}$ R. Lopes De Sá, ${ }^{134}$ J. Lykken, ${ }^{134}$ K. Maeshima, ${ }^{134}$ J. M. Marraffino, ${ }^{134}$ S. Maruyama, ${ }^{134}$ D. Mason, ${ }^{134}$ P. McBride, ${ }^{134}$ P. Merkel, ${ }^{134}$ S. Mrenna, ${ }^{134}$ S. Nahn,,${ }^{134}$ C. Newman-Holmes, ${ }^{134, a}$ V. O'Dell, ${ }^{134}$ K. Pedro ${ }^{134}$ O. Prokofyev, ${ }^{134}$ G. Rakness,${ }^{134}$ E. Sexton-Kennedy, ${ }^{134}$ A. Soha, ${ }^{134}$ W. J. Spalding, ${ }^{134}$ L. Spiegel, ${ }^{134}$ S. Stoynev, ${ }^{134}$ N. Strobbe, ${ }^{134}$ L. Taylor, ${ }^{134}$ S. Tkaczyk, ${ }^{134}$ N. V. Tran,${ }^{134}$ L. Uplegger, ${ }^{134}$ E. W. Vaandering, ${ }^{134}$ C. Vernieri, ${ }^{134}$ M. Verzocchi, ${ }^{134}$ R. Vidal, ${ }^{134}$ M. Wang, ${ }^{134}$ H. A. Weber, ${ }^{134}$ A. Whitbeck, ${ }^{134}$ D. Acosta, ${ }^{135}$ P. Avery, ${ }^{135}$ P. Bortignon, ${ }^{135}$ D. Bourilkov, ${ }^{135}$ A. Carnes, ${ }^{135}$ M. Carver, ${ }^{135}$ D. Curry, ${ }^{135}$ S. Das, ${ }^{135}$ R. D. Field, ${ }^{135}$ I. K. Furic, ${ }^{135}$ S. V. Gleyzer, ${ }^{135}$ J. Konigsberg, ${ }^{135}$ A. Korytov, ${ }^{135}$ K. Kotov, ${ }^{135}$ P. Ma,${ }^{135}$ K. Matchev, ${ }^{135}$ H. Mei, ${ }^{135}$ P. Milenovic, ${ }^{135, n n n}$ G. Mitselmakher, ${ }^{135}$ D. Rank, ${ }^{135}$ R. Rossin, ${ }^{135}$ L. Shchutska, ${ }^{135}$ M. Snowball, ${ }^{135}$ D. Sperka, ${ }^{135}$ N. Terentyev, ${ }^{135}$ L. Thomas, ${ }^{135}$ J. Wang, ${ }^{135}$ S. Wang, ${ }^{135}$ J. Yelton, ${ }^{135}$ S. Hewamanage, ${ }^{136}$ S. Linn, ${ }^{136}$ P. Markowitz, ${ }^{136}$ G. Martinez, ${ }^{136}$ J. L. Rodriguez, ${ }^{136}$ A. Ackert, ${ }^{137}$ J. R. Adams ${ }^{137}$ T. Adams ${ }^{137}$ A. Askew, ${ }^{137}$ S. Bein, ${ }^{137}$ J. Bochenek, ${ }^{137}$ B. Diamond, ${ }^{137}$ J. Haas,${ }^{137}$ S. Hagopian, ${ }^{137}$ V. Hagopian, ${ }^{137}$ K. F. Johnson, ${ }^{137}$ A. Khatiwada, ${ }^{137}$ H. Prosper, ${ }^{137}$ M. Weinberg, ${ }^{137}$ M. M. Baarmand ${ }^{138}$ V. Bhopatkar, ${ }^{138}$ S. Colafranceschi, ${ }^{138,000}$ M. Hohlmann, ${ }^{138}$ H. Kalakhety, ${ }^{138}$ D. Noonan, ${ }^{138}$ T. Roy, ${ }^{138}$ F. Yumiceva, ${ }^{138}$ M. R. Adams, ${ }^{139}$ L. Apanasevich, ${ }^{139}$ D. Berry, ${ }^{139}$ R. R. Betts, ${ }^{139}$ I. Bucinskaite, ${ }^{139}$ R. Cavanaugh, ${ }^{139}$ O. Evdokimov, ${ }^{139}$ L. Gauthier,${ }^{139}$ C. E. Gerber, ${ }^{139}$ D. J. Hofman,${ }^{139}$ P. Kurt, ${ }^{139}$ C. O’Brien, ${ }^{139}$ I. D. Sandoval Gonzalez, ${ }^{139}$ P. Turner, ${ }^{139}$ N. Varelas, ${ }^{139}$ Z. Wu, ${ }^{139}$ M. Zakaria, ${ }^{139}$ B. Bilki, ${ }^{140, p p p}$ W. Clarida, ${ }^{140}$ K. Dilsiz, ${ }^{140}$ S. Durgut, ${ }^{140}$ R. P. Gandrajula, ${ }^{140}$ M. Haytmyradov, ${ }^{140}$ V. Khristenko, ${ }^{140}$ J.-P. Merlo, ${ }^{140}$ H. Mermerkaya, ${ }^{140, q q q}$ A. Mestvirishvili, ${ }^{140}$ A. Moeller, ${ }^{140}$ J. Nachtman, ${ }^{140}$ H. Ogul, ${ }^{140}$ Y. Onel, ${ }^{140}$ F. Ozok, ${ }^{140, r r r}$ A. Penzo, ${ }^{140}$ C. Snyder, ${ }^{140}$ E. Tiras, ${ }^{140}$ J. Wetzel,${ }^{140}$ K. Yi,${ }^{140}$ I. Anderson, ${ }^{141}$ B. A. Barnett, ${ }^{141}$ B. Blumenfeld, ${ }^{141}$ N. Eminizer, ${ }^{141}$ D. Fehling, ${ }^{141}$ L. Feng, ${ }^{141}$ A. V. Gritsan, ${ }^{141}$ P. Maksimovic, ${ }^{141}$ C. Martin, ${ }^{141}$ M. Osherson, ${ }^{141}$ J. Roskes, ${ }^{141}$ A. Sady, ${ }^{141}$ U. Sarica, ${ }^{141}$ M. Swartz, ${ }^{141}$ M. Xiao, ${ }^{141}$ Y. Xin, ${ }^{141}$ C. You, ${ }^{141}$ P. Baringer, ${ }^{142}$ A. Bean, ${ }^{142}$ G. Benelli, ${ }^{142}$ C. Bruner, ${ }^{142}$ R. P. Kenny III, ${ }^{142}$ D. Majumder, ${ }^{142}$ M. Malek, ${ }^{142}$ M. Murray, ${ }^{142}$ S. Sanders, ${ }^{142}$ R. Stringer, ${ }^{142}$ Q. Wang, ${ }^{142}$ A. Ivanov, ${ }^{143}$ K. Kaadze,${ }^{143}$ S. Khalil,${ }^{143}$ M. Makouski, ${ }^{143}$ Y. Maravin, ${ }^{143}$ A. Mohammadi, ${ }^{143}$ L. K. Saini, ${ }^{143}$ N. Skhirtladze, ${ }^{143}$ S. Toda,${ }^{143}$ D. Lange, ${ }^{144}$ F. Rebassoo, ${ }^{144}$ D. Wright, ${ }^{144}$ C. Anelli, ${ }^{145}$ A. Baden, ${ }^{145}$ O. Baron, ${ }^{145}$ A. Belloni, ${ }^{145}$ B. Calvert, ${ }^{145}$ S. C. Eno, ${ }^{145}$ C. Ferraioli, ${ }^{145}$ J. A. Gomez, ${ }^{145}$ N. J. Hadley, ${ }^{145}$ S. Jabeen, ${ }^{145}$ R. G. Kellogg, ${ }^{145}$ T. Kolberg, ${ }^{145}$ J. Kunkle, ${ }^{145}$ Y. Lu, ${ }^{145}$ A. C. Mignerey, ${ }^{145}$ Y. H. Shin, ${ }^{145}$ A. Skuja, ${ }^{145}$ M. B. Tonjes, ${ }^{145}$ S. C. Tonwar, ${ }^{145}$ A. Apyan, ${ }^{146}$ R. Barbieri, ${ }^{146}$ A. Baty, ${ }^{146}$ K. Bierwagen, ${ }^{146}$ S. Brandt, ${ }^{146}$ W. Busza, ${ }^{146}$ I. A. Cali, ${ }^{146}$ Z. Demiragli, ${ }^{146}$ L. Di Matteo, ${ }^{146}$ G. Gomez Ceballos, ${ }^{146}$ M. Goncharov, ${ }^{146}$ D. Gulhan, ${ }^{146}$ Y. Iiyama, ${ }^{146}$ G. M. Innocenti, ${ }^{146}$ M. Klute, ${ }^{146}$ D. Kovalskyi, ${ }^{146}$ Y. S. Lai, ${ }^{146}$ Y.-J. Lee, ${ }^{146}$ A. Levin, ${ }^{146}$ P. D. Luckey, ${ }^{146}$ A. C. Marini, ${ }^{146}$ C. Mcginn, ${ }^{146}$ 
C. Mironov, ${ }^{146}$ S. Narayanan, ${ }^{146}$ X. Niu, ${ }^{146}$ C. Paus, ${ }^{146}$ C. Roland, ${ }^{146}$ G. Roland, ${ }^{146}$ J. Salfeld-Nebgen, ${ }^{146}$ G. S. F. Stephans,${ }^{146}$ K. Sumorok,${ }^{146}$ M. Varma, ${ }^{146}$ D. Velicanu, ${ }^{146}$ J. Veverka, ${ }^{146}$ J. Wang, ${ }^{146}$ T. W. Wang, ${ }^{146}$ B. Wyslouch, ${ }^{146}$ M. Yang, ${ }^{146}$ V. Zhukova, ${ }^{146}$ B. Dahmes, ${ }^{147}$ A. Evans, ${ }^{147}$ A. Finkel, ${ }^{147}$ A. Gude,${ }^{147}$ P. Hansen, ${ }^{147}$ S. Kalafut, ${ }^{147}$ S. C. Kao, ${ }^{147}$ K. Klapoetke, ${ }^{147}$ Y. Kubota, ${ }^{147}$ Z. Lesko, ${ }^{147}$ J. Mans, ${ }^{147}$ S. Nourbakhsh, ${ }^{147}$ N. Ruckstuhl,${ }^{147}$ R. Rusack, ${ }^{147}$ N. Tambe,${ }^{147}$ J. Turkewitz, ${ }^{147}$ J. G. Acosta, ${ }^{148}$ S. Oliveros, ${ }^{148}$ E. Avdeeva, ${ }^{149}$ R. Bartek, ${ }^{149}$ K. Bloom, ${ }^{149}$ S. Bose, ${ }^{149}$ D. R. Claes, ${ }^{149}$ A. Dominguez, ${ }^{149}$ C. Fangmeier, ${ }^{149}$ R. Gonzalez Suarez,${ }^{149}$ R. Kamalieddin,${ }^{149}$ D. Knowlton, ${ }^{149}$ I. Kravchenko, ${ }^{149}$ F. Meier ${ }^{149}$ J. Monroy ${ }^{149}$ F. Ratnikov, ${ }^{149}$ J.E. Siado, ${ }^{149}$ G. R. Snow, ${ }^{149}$ M. Alyari, ${ }^{150}$ J. Dolen, ${ }^{150}$ J. George,${ }^{150}$ A. Godshalk, ${ }^{150}$ C. Harrington, ${ }^{150}$ I. Iashvili, ${ }^{150}$ J. Kaisen, ${ }^{150}$ A. Kharchilava, ${ }^{150}$ A. Kumar, ${ }^{150}$ S. Rappoccio, ${ }^{150}$ B. Roozbahani, ${ }^{150}$ G. Alverson, ${ }^{151}$ E. Barberis, ${ }^{151}$ D. Baumgartel, ${ }^{151}$ M. Chasco,${ }^{151}$ A. Hortiangtham, ${ }^{151}$ A. Massironi, ${ }^{151}$ D. M. Morse,${ }^{151}$ D. Nash, ${ }^{151}$ T. Orimoto, ${ }^{151}$ R. Teixeira De Lima,${ }^{151}$ D. Trocino, ${ }^{151}$ R.-J. Wang, ${ }^{151}$ D. Wood, ${ }^{151}$ J. Zhang, ${ }^{151}$ S. Bhattacharya, ${ }^{152}$ K. A. Hahn, ${ }^{152}$ A. Kubik, ${ }^{152}$ J. F. Low, ${ }^{152}$ N. Mucia, ${ }^{152}$ N. Odell, ${ }^{152}$ B. Pollack, ${ }^{152}$ M. Schmitt,,${ }^{152}$ K. Sung, ${ }^{152}$ M. Trovato, ${ }^{152}$ M. Velasco, ${ }^{152}$ A. Brinkerhoff, ${ }^{153}$ N. Dev, ${ }^{153}$ M. Hildreth, ${ }^{153}$ C. Jessop, ${ }^{153}$ D. J. Karmgard, ${ }^{153}$ N. Kellams,${ }^{153}$ K. Lannon, ${ }^{153}$ N. Marinelli, ${ }^{153}$ F. Meng,${ }^{153}$ C. Mueller, ${ }^{153}$ Y. Musienko, ${ }^{153, \mathrm{~mm}}$ M. Planer,${ }^{153}$ A. Reinsvold, ${ }^{153}$ R. Ruchti, ${ }^{153}$ G. Smith, ${ }^{153}$ S. Taroni, ${ }^{153}$ N. Valls ${ }^{153}$ M. Wayne, ${ }^{153}$ M. Wolf, ${ }^{153}$ A. Woodard, ${ }^{153}$ L. Antonelli, ${ }^{154}$ J. Brinson, ${ }^{154}$ B. Bylsma, ${ }^{154}$ L. S. Durkin, ${ }^{154}$ S. Flowers, ${ }^{154}$ A. Hart, ${ }^{154}$ C. Hill,${ }^{154}$ R. Hughes, ${ }^{154}$ W. Ji, ${ }^{154}$ T. Y. Ling, ${ }^{154}$ B. Liu, ${ }^{154}$ W. Luo, ${ }^{154}$ D. Puigh, ${ }^{154}$ M. Rodenburg, ${ }^{154}$ B. L. Winer, ${ }^{154}$ H. W. Wulsin, ${ }^{154}$ O. Driga,${ }^{155}$ P. Elmer, ${ }^{155}$ J. Hardenbrook, ${ }^{155}$ P. Hebda, ${ }^{155}$ S. A. Koay, ${ }^{155}$ P. Lujan, ${ }^{155}$ D. Marlow, ${ }^{155}$ T. Medvedeva,${ }^{155}$ M. Mooney, ${ }^{155}$ J. Olsen, ${ }^{155}$ C. Palmer, ${ }^{155}$ P. Piroué, ${ }^{155}$ H. Saka, ${ }^{155}$ D. Stickland, ${ }^{155}$ C. Tully, ${ }^{155}$ A. Zuranski, ${ }^{155}$ S. Malik, ${ }^{156}$ A. Barker, ${ }^{157}$ V. E. Barnes,${ }^{157}$ D. Benedetti, ${ }^{157}$ D. Bortoletto, ${ }^{157}$ L. Gutay, ${ }^{157}$ M. K. Jha, ${ }^{157}$ M. Jones, ${ }^{157}$ A. W. Jung, ${ }^{157}$ K. Jung, ${ }^{157}$ A. Kumar,${ }^{157}$ D. H. Miller ${ }^{157}$ N. Neumeister, ${ }^{157}$ B. C. Radburn-Smith, ${ }^{157}$ X. Shi, ${ }^{157}$ I. Shipsey, ${ }^{157}$ D. Silvers, ${ }^{157}$ J. Sun, ${ }^{157}$ A. Svyatkovskiy, ${ }^{157}$ F. Wang, ${ }^{157}$ W. Xie, ${ }^{157}$ L. Xu, ${ }^{157}$ N. Parashar, ${ }^{158}$ J. Stupak, ${ }^{158}$ A. Adair, ${ }^{159}$ B. Akgun, ${ }^{159}$ Z. Chen, ${ }^{159}$ K. M. Ecklund, ${ }^{159}$ F. J. M. Geurts, ${ }^{159}$ M. Guilbaud ${ }^{159}$ W. Li ${ }^{159}$ B. Michlin, ${ }^{159}$ M. Northup,${ }^{159}$ B. P. Padley,${ }^{159}$ R. Redjimi, ${ }^{159}$ J. Roberts, ${ }^{159}$ J. Rorie, ${ }^{159}$ Z. Tu, ${ }^{159}$ J. Zabel, ${ }^{159}$ B. Betchart, ${ }^{160}$ A. Bodek, ${ }^{160}$ P. de Barbaro, ${ }^{160}$ R. Demina, ${ }^{160}$ Y. Eshaq, ${ }^{160}$

T. Ferbel, ${ }^{160}$ M. Galanti, ${ }^{160}$ A. Garcia-Bellido, ${ }^{160}$ J. Han, ${ }^{160}$ A. Harel, ${ }^{160}$ O. Hindrichs, ${ }^{160}$ A. Khukhunaishvili, ${ }^{160}$ G. Petrillo, ${ }^{160}$ P. Tan, ${ }^{160}$ M. Verzetti, ${ }^{160}$ J. P. Chou, ${ }^{161}$ E. Contreras-Campana, ${ }^{161}$ D. Ferencek, ${ }^{161}$ Y. Gershtein, ${ }^{161}$ E. Halkiadakis, ${ }^{161}$ D. Hidas, ${ }^{161}$ E. Hughes,${ }^{161}$ S. Kaplan, ${ }^{161}$ R. Kunnawalkam Elayavalli, ${ }^{161}$ A. Lath, ${ }^{161}$ K. Nash, ${ }^{161}$ S. Salur, ${ }^{161}$ S. Schnetzer, ${ }^{161}$ D. Sheffield, ${ }^{161}$ S. Somalwar, ${ }^{161}$ R. Stone, ${ }^{161}$ S. Thomas, ${ }^{161}$ P. Thomassen, ${ }^{161}$ M. Walker, ${ }^{161}$ M. Foerster, ${ }^{162}$ G. Riley, ${ }^{162}$ K. Rose, ${ }^{162}$ S. Spanier, ${ }^{162}$ O. Bouhali, ${ }^{163, \text { sss }}$ A. Castaneda Hernandez, ${ }^{163, \text { sss }}$ A. Celik, ${ }^{163}$ M. Dalchenko, ${ }^{163}$ M. De Mattia ${ }^{163}$ A. Delgado, ${ }^{163}$ S. Dildick, ${ }^{163}$ R. Eusebi, ${ }^{163}$ J. Gilmore, ${ }^{163}$ T. Huang, ${ }^{163}$ T. Kamon, ${ }^{163, t t t}$ V. Krutelyov, ${ }^{163}$ R. Mueller, ${ }^{163}$ I. Osipenkov, ${ }^{163}$ Y. Pakhotin, ${ }^{163}$ R. Patel, ${ }^{163}$ A. Perloff, ${ }^{163}$ A. Rose, ${ }^{163}$ A. Safonov, ${ }^{163}$ A. Tatarinov, ${ }^{163}$ K. A. Ulmer, ${ }^{163, c}$ N. Akchurin,,${ }^{164}$ C. Cowden,,${ }^{164}$ J. Damgov, ${ }^{164}$ C. Dragoiu, ${ }^{164}$ P. R. Dudero, ${ }^{164}$ J. Faulkner, ${ }^{164}$ S. Kunori, ${ }^{164}$ K. Lamichhane, ${ }^{164}$ S. W. Lee, ${ }^{164}$ T. Libeiro, ${ }^{164}$ S. Undleeb, ${ }^{164}$ I. Volobouev, ${ }^{164}$ E. Appelt, ${ }^{165}$

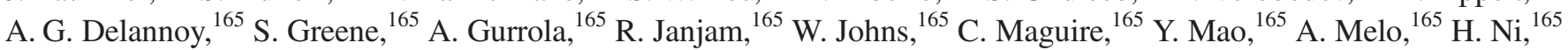
P. Sheldon, ${ }^{165}$ S. Tuo, ${ }^{165}$ J. Velkovska, ${ }^{165}$ Q. Xu,${ }^{165}$ M. W. Arenton, ${ }^{166}$ B. Cox, ${ }^{166}$ B. Francis, ${ }^{166}$ J. Goodell, ${ }^{166}$ R. Hirosky, ${ }^{166}$ A. Ledovskoy, ${ }^{166} \mathrm{H}$. Li, ${ }^{166}$ C. Lin, ${ }^{166}$ C. Neu, ${ }^{166}$ T. Sinthuprasith, ${ }^{166}$ X. Sun, ${ }^{166}$ Y. Wang, ${ }^{166}$ E. Wolfe, ${ }^{166}$ J. Wood, ${ }^{166}$ F. Xia, ${ }^{166}$ C. Clarke,${ }^{167}$ R. Harr, ${ }^{167}$ P. E. Karchin, ${ }^{167}$ C. Kottachchi Kankanamge Don, ${ }^{167}$ P. Lamichhane, ${ }^{167}$ J. Sturdy, ${ }^{167}$ D. A. Belknap, ${ }^{168}$ D. Carlsmith, ${ }^{168}$ M. Cepeda, ${ }^{168}$ S. Dasu, ${ }^{168}$ L. Dodd, ${ }^{168}$ S. Duric, ${ }^{168}$ B. Gomber, ${ }^{168}$ M. Grothe, ${ }^{168}$ R. Hall-Wilton, ${ }^{168}$ M. Herndon, ${ }^{168}$ A. Hervé, ${ }^{168}$ P. Klabbers, ${ }^{168}$ A. Lanaro, ${ }^{168}$ A. Levine, ${ }^{168}$ K. Long, ${ }^{168}$ R. Loveless, ${ }^{168}$ A. Mohapatra, ${ }^{168}$ I. Ojalvo, ${ }^{168}$ T. Perry, ${ }^{168}$ G. A. Pierro, ${ }^{168}$ G. Polese, ${ }^{168}$ T. Ruggles, ${ }^{168}$ T. Sarangi, ${ }^{168}$ A. Savin, ${ }^{168}$ A. Sharma, ${ }^{168}$ N. Smith, ${ }^{168}$ W. H. Smith, ${ }^{168}$ D. Taylor, ${ }^{168}$ P. Verwilligen, ${ }^{168}$ and N. Woods ${ }^{168}$

\title{
(CMS Collaboration)
}

\author{
${ }^{1}$ Yerevan Physics Institute, Yerevan, Armenia \\ ${ }^{2}$ Institut für Hochenergiephysik der OeAW, Wien, Austria \\ ${ }^{3}$ National Centre for Particle and High Energy Physics, Minsk, Belarus \\ ${ }^{4}$ Universiteit Antwerpen, Antwerpen, Belgium \\ ${ }^{5}$ Vrije Universiteit Brussel, Brussel, Belgium \\ ${ }^{6}$ Université Libre de Bruxelles, Bruxelles, Belgium \\ ${ }^{7}$ Ghent University, Ghent, Belgium \\ ${ }^{8}$ Université Catholique de Louvain, Louvain-la-Neuve, Belgium
}




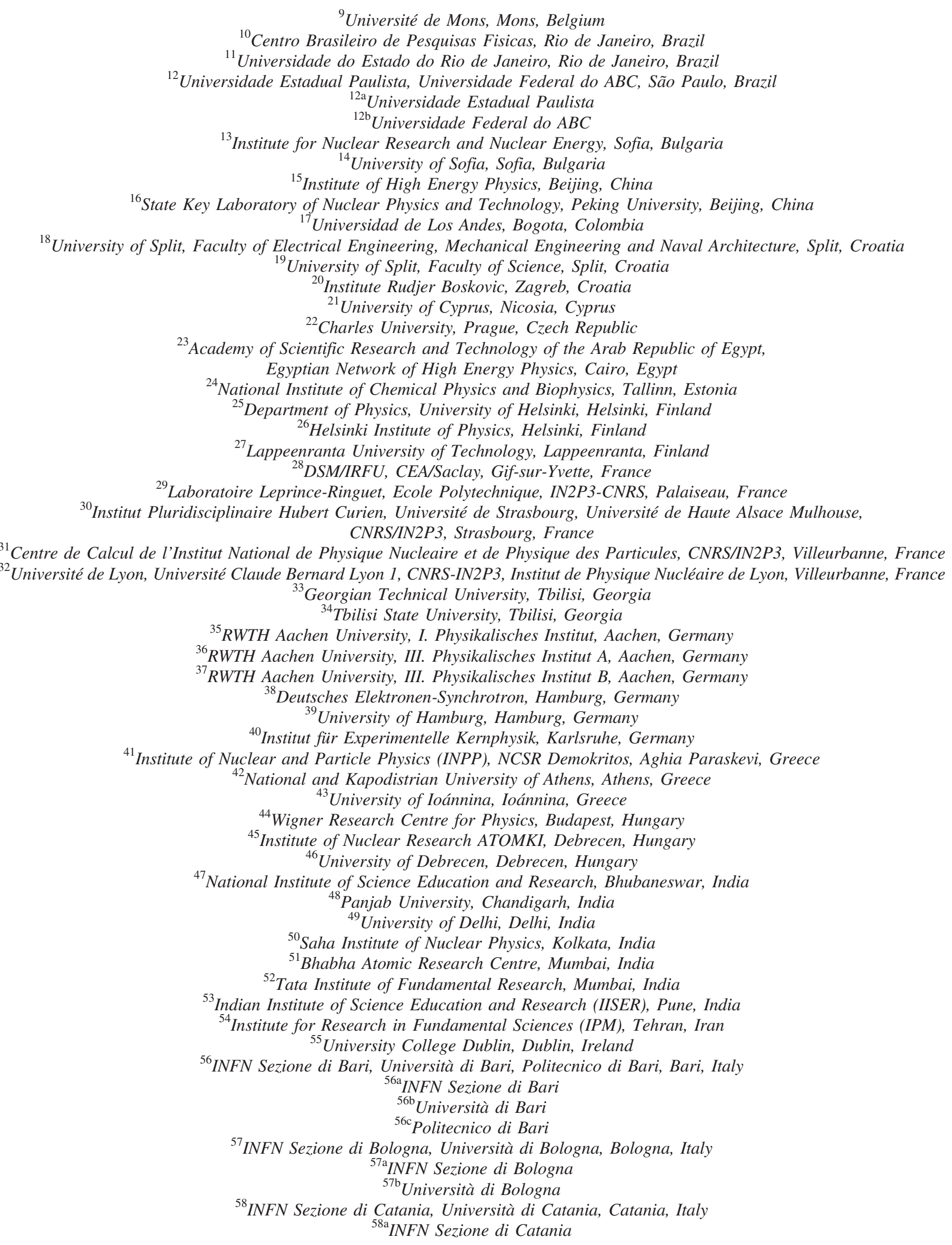


${ }^{58 \mathrm{~b}}$ Università di Catania

${ }^{59}$ INFN Sezione di Firenze, Università di Firenze, Firenze, Italy

${ }^{59 a}$ INFN Sezione di Firenze

${ }^{59 \mathrm{~b}}$ Università di Firenze

${ }^{60}$ INFN Laboratori Nazionali di Frascati, Frascati, Italy

${ }^{61}$ INFN Sezione di Genova, Università di Genova, Genova, Italy

${ }^{61 \mathrm{a}}$ INFN Sezione di Genova

${ }^{61 \mathrm{~b}}$ Università di Genova

${ }^{62}$ INFN Sezione di Milano-Bicocca, Università di Milano-Bicocca, Milano, Italy

${ }^{62 \mathrm{a}}$ INFN Sezione di Milano-Bicocca

${ }^{62 \mathrm{~b}}$ Università di Milano-Bicocca

${ }^{63}$ INFN Sezione di Napoli, Università di Napoli 'Federico II', Napoli, Italy,

Università della Basilicata, Potenza, Italy, Università G. Marconi, Roma, Italy

${ }^{63 \mathrm{a}}$ INFN Sezione di Napoli

${ }^{63 \mathrm{~b}}$ Università di Napoli 'Federico II'

${ }^{63 \mathrm{c}}$ Università della Basilicata

${ }^{63 \mathrm{~d}}$ Università G. Marconi

${ }^{64}$ INFN Sezione di Padova, Università di Padova, Padova, Italy, Università di Trento, Trento, Italy

${ }^{64 a}$ INFN Sezione di Padova

${ }^{64 \mathrm{~b}}$ Università di Padova

${ }^{64 \mathrm{c}}$ Università di Trento

${ }^{65}$ INFN Sezione di Pavia, Università di Pavia, Pavia, Italy

${ }^{65 a}$ INFN Sezione di Pavia

${ }^{65 \mathrm{~b}}$ Università di Pavia

${ }^{66}$ INFN Sezione di Perugia, Università di Perugia, Perugia, Italy

${ }^{66 a}$ INFN Sezione di Perugia

${ }^{66 \mathrm{~b}}$ Università di Perugia

${ }^{67}$ INFN Sezione di Pisa, Università di Pisa, Scuola Normale Superiore di Pisa, Pisa, Italy

${ }^{67 a}$ INFN Sezione di Pisa

${ }^{67 \mathrm{~b}}$ Università di Pisa

${ }^{67 \mathrm{c}}$ Scuola Normale Superiore di Pisa

${ }^{68 \mathrm{a}}$ INFN Sezione di Roma

${ }^{68 \mathrm{~b}}$ Università di Roma

${ }^{69}$ INFN Sezione di Torino, Università di Torino, Torino, Italy, Università del Piemonte Orientale, Novara, Italy

${ }^{69}$ a INFN Sezione di Torino

${ }^{69 \mathrm{~b}}$ Università di Torino

${ }^{69 \mathrm{c}}$ Università del Piemonte Orientale

${ }^{70}$ INFN Sezione di Trieste, Università di Trieste, Trieste, Italy

${ }^{70 a}$ INFN Sezione di Trieste

${ }^{70 \mathrm{~b}}$ Università di Trieste

${ }^{71}$ Kangwon National University, Chunchon, Korea

${ }^{72}$ Kyungpook National University, Daegu, Korea

${ }^{73}$ Chonbuk National University, Jeonju, Korea

${ }^{74}$ Chonnam National University, Institute for Universe and Elementary Particles, Kwangju, Korea

${ }^{75}$ Korea University, Seoul, Korea

${ }^{76}$ Seoul National University, Seoul, Korea

${ }^{77}$ University of Seoul, Seoul, Korea

${ }^{78}$ Sungkyunkwan University, Suwon, Korea

${ }^{79}$ Vilnius University, Vilnius, Lithuania

${ }^{80}$ National Centre for Particle Physics, Universiti Malaya, Kuala Lumpur, Malaysia

${ }^{81}$ Centro de Investigacion y de Estudios Avanzados del IPN, Mexico City, Mexico

${ }^{82}$ Universidad Iberoamericana, Mexico City, Mexico

${ }^{83}$ Benemerita Universidad Autonoma de Puebla, Puebla, Mexico

${ }^{84}$ Universidad Autónoma de San Luis Potosí, San Luis Potosí, Mexico

${ }^{85}$ University of Auckland, Auckland, New Zealand

${ }^{86}$ University of Canterbury, Christchurch, New Zealand

${ }^{87}$ National Centre for Physics, Quaid-I-Azam University, Islamabad, Pakistan

${ }^{88}$ National Centre for Nuclear Research, Swierk, Poland

${ }^{89}$ Institute of Experimental Physics, Faculty of Physics, University of Warsaw, Warsaw, Poland

${ }^{90}$ Laboratório de Instrumentação e Física Experimental de Partículas, Lisboa, Portugal 


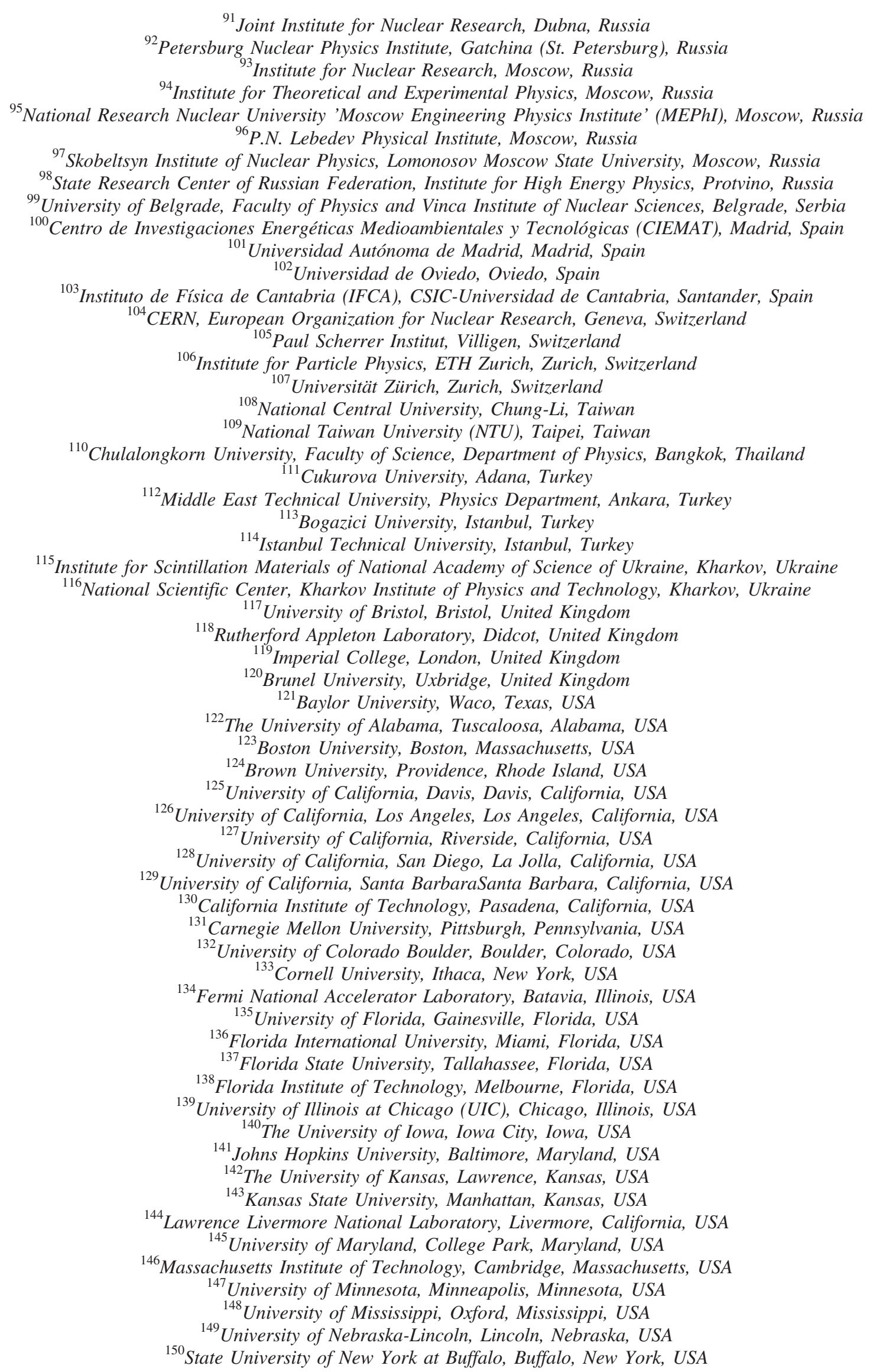




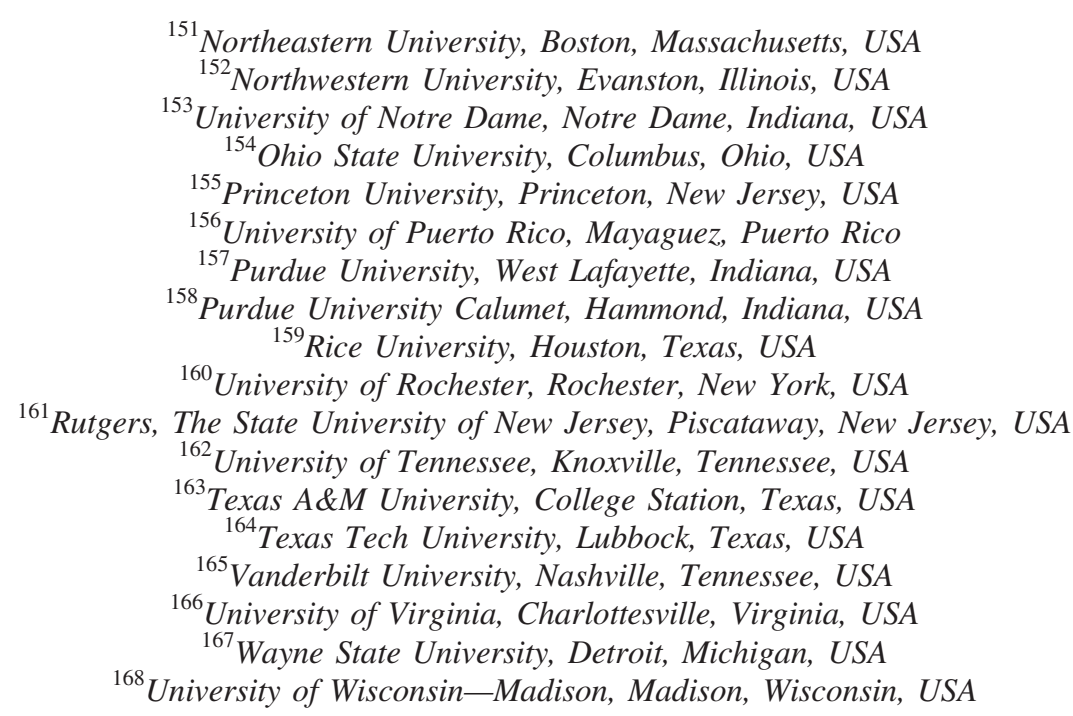

${ }^{\mathrm{a}}$ Deceased.

${ }^{\mathrm{b}}$ Also at Vienna University of Technology, Vienna, Austria.

${ }^{c}$ Also at CERN, European Organization for Nuclear Research, Geneva, Switzerland.

${ }^{\mathrm{d}}$ Also at State Key Laboratory of Nuclear Physics and Technology, Peking University, Beijing, China.

${ }^{\mathrm{e}}$ Also at Institut Pluridisciplinaire Hubert Curien, Université de Strasbourg, Université de Haute Alsace Mulhouse, CNRS/IN2P3, Strasbourg, France.

${ }^{\mathrm{f}}$ Also at National Institute of Chemical Physics and Biophysics, Tallinn, Estonia.

${ }^{\mathrm{g}}$ Also at Skobeltsyn Institute of Nuclear Physics, Lomonosov Moscow State University, Moscow, Russia.

${ }^{\mathrm{h}}$ Also at Universidade Estadual de Campinas, Campinas, Brazil.

${ }^{\mathrm{i}}$ Also at Centre National de la Recherche Scientifique (CNRS) - IN2P3, Paris, France.

${ }^{\mathrm{j}}$ Also at Laboratoire Leprince-Ringuet, Ecole Polytechnique, IN2P3-CNRS, Palaiseau, France.

${ }^{\mathrm{k}}$ Also at Joint Institute for Nuclear Research, Dubna, Russia.

${ }^{1}$ Also at British University in Egypt, Cairo, Egypt.

${ }^{\mathrm{m}}$ Also at Suez University, Suez, Egypt.

${ }^{\mathrm{n}}$ Also at Cairo University, Cairo, Egypt.

${ }^{\circ}$ Also at Fayoum University, El-Fayoum, Egypt.

${ }^{\mathrm{p}}$ Also at Université de Haute Alsace, Mulhouse, France.

${ }^{\mathrm{q}}$ Also at Tbilisi State University, Tbilisi, Georgia.

${ }^{\mathrm{r}}$ Also at RWTH Aachen University, III. Physikalisches Institut A, Aachen, Germany.

${ }^{s}$ Also at University of Hamburg, Hamburg, Germany.

${ }^{\mathrm{t}}$ Also at Brandenburg University of Technology, Cottbus, Germany.

${ }^{u}$ Also at Institute of Nuclear Research ATOMKI, Debrecen, Hungary.

${ }^{v}$ Also at Eötvös Loránd University, Budapest, Hungary.

${ }^{\mathrm{w}}$ Also at University of Debrecen, Debrecen, Hungary.

${ }^{\mathrm{x}}$ Also at Wigner Research Centre for Physics, Budapest, Hungary.

${ }^{y}$ Also at Indian Institute of Science Education and Research, Bhopal, India.

${ }^{\mathrm{z}}$ Also at University of Visva-Bharati, Santiniketan, India.

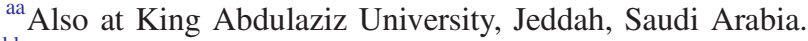

${ }^{\mathrm{bb}}$ Also at University of Ruhuna, Matara, Sri Lanka.

${ }^{\mathrm{cc}}$ Also at Isfahan University of Technology, Isfahan, Iran.

${ }^{\mathrm{dd}}$ Also at University of Tehran, Department of Engineering Science, Tehran, Iran.

${ }^{e e}$ Also at Plasma Physics Research Center, Science and Research Branch, Islamic Azad University, Tehran, Iran.

${ }^{\mathrm{ff}}$ Also at Laboratori Nazionali di Legnaro dell'INFN, Legnaro, Italy.

${ }^{g g}$ Also at Università degli Studi di Siena, Siena, Italy.

${ }^{\text {hh }}$ Also at Purdue University, West Lafayette, USA.

${ }^{\text {ii } A l s o ~ a t ~ I n t e r n a t i o n a l ~ I s l a m i c ~ U n i v e r s i t y ~ o f ~ M a l a y s i a, ~ K u a l a ~ L u m p u r, ~ M a l a y s i a . ~}$

${ }^{\mathrm{jj}}$ Also at Malaysian Nuclear Agency, MOSTI, Kajang, Malaysia.

${ }^{\mathrm{kk}}$ Also at Consejo Nacional de Ciencia y Tecnología, Mexico city, Mexico.

${ }^{11}$ Also at Warsaw University of Technology, Institute of Electronic Systems, Warsaw, Poland.

${ }^{\mathrm{mm}}$ Also at Institute for Nuclear Research, Moscow, Russia.

${ }^{n n}$ Also at National Research Nuclear University 'Moscow Engineering Physics Institute' (MEPhI), Moscow, Russia. 


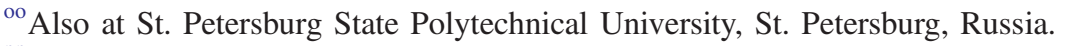

${ }^{\mathrm{pp}}$ Also at California Institute of Technology, Pasadena, USA.

${ }^{\mathrm{qq}}$ Also at Faculty of Physics, University of Belgrade, Belgrade, Serbia.

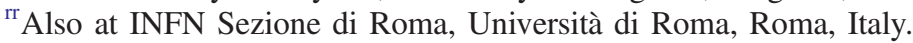

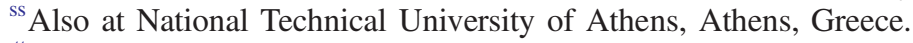

"Also at Scuola Normale e Sezione dell'INFN, Pisa, Italy.

${ }^{u u}$ Also at National and Kapodistrian University of Athens, Athens, Greece.

${ }^{\mathrm{vv}}$ Also at Institute for Theoretical and Experimental Physics, Moscow, Russia.

${ }^{w w}$ Also at Albert Einstein Center for Fundamental Physics, Bern, Switzerland.

${ }^{\mathrm{xx}}$ Also at Gaziosmanpasa University, Tokat, Turkey.

${ }^{\text {yy }}$ Also at Mersin University, Mersin, Turkey.

${ }^{\mathrm{zz}}$ Also at Cag University, Mersin, Turkey.

${ }^{\text {aaa }}$ Also at Piri Reis University, Istanbul, Turkey.

${ }^{b b b}$ Also at Adiyaman University, Adiyaman, Turkey.

${ }^{c c c}$ Also at Ozyegin University, Istanbul, Turkey.

${ }^{\text {ddd }}$ Also at Izmir Institute of Technology, Izmir, Turkey.

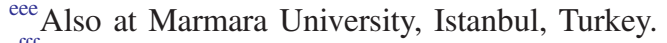

${ }^{\mathrm{fff}}$ Also at Kafkas University, Kars, Turkey.

${ }^{\text {ggg }}$ Also at Istanbul Bilgi University, Istanbul, Turkey.

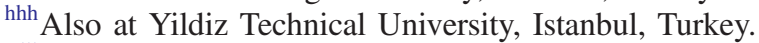

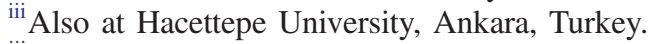

${ }^{\mathrm{jjj}}$ Also at Rutherford Appleton Laboratory, Didcot, United Kingdom.

${ }^{k k k}$ Also at School of Physics and Astronomy, University of Southampton, Southampton, United Kingdom.

${ }^{111}$ Also at Instituto de Astrofísica de Canarias, La Laguna, Spain.

${ }^{\mathrm{mmm}}$ Also at Utah Valley University, Orem, USA.

${ }^{n n n}$ Also at University of Belgrade, Faculty of Physics and Vinca Institute of Nuclear Sciences, Belgrade, Serbia.

${ }^{000}$ Also at Facoltà Ingegneria, Università di Roma, Roma, Italy.

${ }^{\mathrm{ppp}}$ Also at Argonne National Laboratory, Argonne, USA.

${ }^{\text {qqq }}$ Also at Erzincan University, Erzincan, Turkey.

${ }^{\mathrm{rrr}}$ Also at Mimar Sinan University, Istanbul, Istanbul, Turkey.

${ }^{\text {sss }}$ Also at Texas A\&M University at Qatar, Doha, Qatar.

${ }^{t t t}$ Also at Kyungpook National University, Daegu, Korea. 